\title{
FRACTIONAL NLS EQUATIONS WITH MAGNETIC FIELD, CRITICAL FREQUENCY AND CRITICAL GROWTH
}

\author{
ZHANG BINLIN, MARCO SQUASSINA, AND ZHANG XIA
}

\begin{abstract}
The paper is devoted to the study of a singularly perturbed fractional Schrödinger equations involving critical frequency and critical growth in the presence of a magnetic field. By using variational methods, we obtain the existence of mountain pass solutions $u_{\varepsilon}$ which tend to the trivial solution as $\varepsilon \rightarrow 0$. Moreover, we get infinitely many solutions and sign-changing solutions for the problem in absence of magnetic effects under some extra assumptions.
\end{abstract}

\section{INTRODUCTION AND MAIN RESULT}

In this paper, we study the following Schrödinger equations involving a critical nonlinearity

$$
\varepsilon^{2 \alpha}(-\Delta)_{A_{\varepsilon}}^{\alpha} u+V(x) u=f(x,|u|) u+K(x)|u|^{2_{\alpha}^{*}-2} u \quad \text { in } \mathbb{R}^{N},
$$

driven by the magnetic fractional Laplacian operator $(-\Delta)_{A_{\varepsilon}}^{\alpha}$ of order $\alpha \in(0,1)$, where $N \geq 2$, $\varepsilon$ is a positive parameter, $2_{\alpha}^{*}=2 N /(N-2 \alpha)$ is the critical Sobolev exponent, $V: \mathbb{R}^{N} \rightarrow \mathbb{R}$ and $A: \mathbb{R}^{N} \rightarrow \mathbb{R}^{N}$ are the electric and magnetic potentials respectively and $A_{\varepsilon}(x):=\varepsilon^{-1} A(x)$. If $A$ is a smooth function, the nonlocal operator $(-\Delta)_{A}^{\alpha}$, which up to normalization constants can be defined on smooth functions $u$ as

$$
(-\Delta)_{A}^{\alpha} u(x):=2 \lim _{\varepsilon \rightarrow 0} \int_{B_{\varepsilon}^{c}(x)} \frac{u(x)-e^{\mathrm{i}(x-y) \cdot A\left(\frac{x+y}{2}\right)} u(y)}{|x-y|^{N+2 \alpha}} d y, \quad x \in \mathbb{R}^{N},
$$

has been recently introduced in [13]. The motivations for its introduction are described in $[13,32]$ in more detail and rely essentially on the Lévy-Khintchine formula for the generator of a general Lévy process. If the magnetic field $A \not \equiv 0$, it seems that the first work which considered the existence of solutions for problem (1.1) in the subcritical case with $\varepsilon=1$, formally $\alpha=1$ and $K=0$ was [16]. For more details on fractional magnetic operators we refer to [19-21] for related physical background. If the magnetic field $A \equiv 0$, the above operator is consistent with the usual notion of fractional Laplacian, which may be viewed as the infinitesimal generators of a Lévy stable diffusion processes (see [1]). This operator arises in the description of various phenomena in the applied sciences, such as phase transitions, materials science, conservation laws, minimal surfaces, water waves, optimization, plasma physics. See [1] and the references therein for a more detailed introduction. Some interesting models involving the fractional Laplacian have received much attention recently, such as the fractional Schrödinger equation (see $[2,8,17,22,23]$ ), the fractional Kirchhoff equation (see $[18,25]$ ) and the fractional porous medium equation (see [33]). Another driving force for the study of problem (1.1) arises in the study of the following timedependent local Schrödinger equation

$$
\mathrm{i} \hbar \frac{\partial \psi}{\partial t}=\frac{1}{2 m}\left(\frac{\hbar}{\mathrm{i}} \nabla-A(x)\right)^{2} \psi+W(x) \psi-g(x,|\psi|) \psi,
$$

where $\hbar$ is the Planck constant, $m$ is the mass of the particle, $A: \mathbb{R}^{N} \rightarrow \mathbb{R}^{N}$ is the magnetic potential, $W: \mathbb{R}^{N} \rightarrow \mathbb{R}^{N}$ is the electric potential, $g$ is the nonlinear coupling and $\psi$ is the wave

2010 Mathematics Subject Classification. Primary 35R11, 35J62, 35B33, Secondary 35A15.

Key words and phrases. Fractional NLS, magnetic operator, critical Sobolev exponent, critical frequency. 
function representing the state of the particle. This equation arises in Quantum Mechanics and describes the dynamics of the particle in a non-relativistic setting, see for example [26]. Clearly, the form $\psi(x, t)=e^{-i \omega t \hbar^{-1}} u(x)$ is a standing wave solution of (1.2) if and only if $u$ satisfies the following stationary equation

$$
\left(\frac{\varepsilon}{\mathrm{i}} \nabla-A(x)\right)^{2} u+V(x) u=f(x,|u|) u .
$$

where $\varepsilon=\hbar, V(x)=2 m(W(x)-\omega), f=2 m g$ and

$$
\left(\frac{\varepsilon}{\mathrm{i}} \nabla-A(x)\right)^{2} u=-\varepsilon^{2} \Delta u-\frac{2 \varepsilon}{\mathrm{i}} A(x) \cdot \nabla u+|A(x)|^{2} u-\frac{\varepsilon}{\mathrm{i}} \operatorname{div} A(x) u .
$$

See [14] and the references cited therein for recent results in this direction (see also [31]). Similarly, we could derive the fractional version of (1.3) as $A=0$ and $\varepsilon=1$, which is a fundamental equation of fractional Quantum Mechanics in the study of particles on stochastic fields modeled by Lévy processes, see [23]. Also we refer the reader to [22] for extended physical description.

Recently, the study on fractional Schrödinger equation has attracted much attention. On the one hand, some recent works involving the subcritical case have been obtained. Felmer et al. in [17] studied the following equations with $A=0$ and $V=1$

$$
(-\Delta)^{\alpha} u+V(x) u=f(x, u) .
$$

Using critical point theory, they obtained the existence of a ground state. Regularity, decay and symmetry properties of these solutions were also analyzed. In [10], Cheng investigated the existence of ground state for (1.5) when $f(x, t)=|t|^{p-2} t$, in which the coercivity assumption $V(x) \rightarrow+\infty$ for $|x| \rightarrow \infty$ is imposed. In [27], by using Mountain Pass arguments and a comparison method, Secchi considered the existence of ground state for (1.5) when the potential $V$ satisfies the assumption $\liminf _{|x| \rightarrow \infty} V(x) \geq V_{\infty}$. In [24], assuming that $V^{-1}(0)$ has nonempty interior, Ledesma obtained the existence of nontrivial solutions and explored the concentration phenomenon of solutions for (1.5). In [9] Chen and Zheng studied the problem

$$
\varepsilon^{2 \alpha}(-\Delta)^{\alpha} u+V(x) u=f(x, u) \quad \text { in } \mathbb{R}^{N},
$$

where $N \leq 3, f(x, t)=|t|^{p-2} t$ and $V(x)$ satisfies some smoothness and boundedness assumptions. By using the Lyapunov-Schmidt reduction method, they showed that (1.6) has a nontrivial solution $u_{\varepsilon}$ concentrating to some single point as $\varepsilon \rightarrow 0$. In [11], assuming that $f(x, t)=$ $|t|^{p-2} t$ and $V$ is a sufficiently smooth potential with $\inf _{\mathbb{R}^{N}} V>0$, Dávila et al. recovered various existence results already known for the case $\alpha=1$ and showed the existence of solutions around $k$ nondegenerate critical points of $V$ for (1.6). In [30], Shang and Zhang studied the concentration phenomenon of solutions for (1.6) under the assumptions $f(x, t)=K(x)|t|^{p-2} t$, $V, K$ are positive smooth functions and $\inf _{\mathbb{R}^{N}} V>0$. By a perturbative methods, they showed existence of solutions which concentrate near some critical points of the function

$$
\Gamma(x)=(V(x))^{\frac{p+2}{p}-\frac{N}{2 \alpha}}(K(x))^{-\frac{2}{p+1}} .
$$

On the other hand, there are some recent papers dedicated to the study of fractional Schrödinger equations with critical growth under various hypotheses on the potential function $V(x)$. In [29], Shang and Zhang studied the existence for the critical fractional Schrödinger equation

$$
\varepsilon^{2 \alpha}(-\Delta)^{\alpha} u+V(x) u=\lambda f(u)+|u|^{2_{\alpha}^{*}-2} u \quad \text { in } \mathbb{R}^{N},
$$

where $0<\inf _{\mathbb{R}^{N}} V<\liminf _{|x| \rightarrow \infty} V(x)<+\infty$. Based on variational methods, they showed that problem (1.7) has a nonnegative ground state solution for all sufficiently large $\lambda$ and small $\varepsilon$. Moreover, Shen and Gao in [28] obtained the existence of nontrivial solutions for problem (1.7) under assumptions that potential function $V$ is nonnegative and trapping, namely $\liminf _{|x| \rightarrow \infty} V(x)=+\infty$. As for the case $\varepsilon=1$, we refer to [34,35] for some recent results. 
Motivated by the above works, especially by [14,15], we are interested in critical fractional Schrödinger equations with the magnetic field and the critical frequency case in the sense that $\min _{\mathbb{R}^{N}} V=0$. It is worth mentioning that the study of fractional Schrödinger equations with the critical frequency was first investigated by Byeon and Wang [4,5]. Main difficulties arise, when dealing with this problem, because of the appearance of the magnetic field and the critical frequency, and of the nonlocal nature of the fractional Laplacian. For this, we need to develop new techniques to overcome difficulties induced by these new features.

We shall assume the following conditions:

$\left(V_{1}\right) V \in C\left(\mathbb{R}^{N}, \mathbb{R}\right)$ and $\min _{\mathbb{R}^{N}} V=0 ;$

$\left(V_{2}\right)$ There exists $a>0$ such that $V^{a}=\left\{x \in \mathbb{R}^{N}: V(x)<a\right\}$ has finite Lebesgue measure;

$(K)$ There exist $K_{0}, K_{1}>0$ such that $K_{0} \leq K(x) \leq K_{1}$ for any $x \in \mathbb{R}^{N}$;

$\left(f_{1}\right) f \in C\left(\mathbb{R}^{N} \times \mathbb{R}^{+}, \mathbb{R}\right)$ and there exists $c_{0}>0$ and $p \in\left(2,2_{\alpha}^{*}\right)$ such that

$$
|f(x, t)| \leq c_{0}\left(1+|t|^{p-2}\right), \quad \text { for any }(x, t) \in \mathbb{R}^{N} \times \mathbb{R}^{+} ;
$$

$\left(f_{2}\right) \lim _{t \rightarrow 0+} f(x, t)=0$ uniformly in $x \in \mathbb{R}^{N}$;

$\left(f_{3}\right)$ There exists $\mu>2$ such that $\mu F(x, t) \leq f(x, t) t^{2}$ for any $t>0, F(x, t):=\int_{0}^{t} f(x, s) s d s$;

$\left(f_{4}\right)$ There exist $c_{1}>0, q \in\left(2,2_{\alpha}^{*}\right)$ such that $f(x, t) \geq c_{1} t^{q-2}$ for any $t>0$.

We say that $u \in X_{\varepsilon}$ is a (weak) solution of problem (1.1) if for any $v \in X_{\varepsilon}$,

$$
\begin{aligned}
\operatorname{Re} \int_{\mathbb{R}^{2 N}} & \frac{\left(u(x)-e^{\mathrm{i}(x-y) \cdot A_{\varepsilon}\left(\frac{x+y}{2}\right)} u(y)\right) \overline{\left(v(x)-e^{\mathrm{i}(x-y) \cdot A_{\varepsilon}\left(\frac{x+y}{2}\right)} v(y)\right)}}{|x-y|^{N+2 \alpha}} d x d y \\
& +\varepsilon^{-2 \alpha} \operatorname{Re} \int_{\mathbb{R}^{N}} V(x) u \bar{v} d x=\varepsilon^{-2 \alpha} \operatorname{Re} \int_{\mathbb{R}^{N}}\left(f(x,|u|) u+K(x)|u|^{2_{\alpha}^{*}-2} u\right) \bar{v} d x .
\end{aligned}
$$

where $\bar{z}$ denotes complex conjugate of $z \in \mathbb{C}$, Rez is the real part of $z,\left(X_{\varepsilon},\|\cdot\|_{X_{\varepsilon}}\right)$ is a suitable subspace of the fractional space $H_{A_{\varepsilon}}^{\alpha}\left(\mathbb{R}^{N}, \mathbb{C}\right)$. See Section 2 for more details.

We are now in a position to state the main result of the paper.

Theorem 1.1. Assume that $\left(V_{1}\right)-\left(V_{2}\right),\left(f_{1}\right)-\left(f_{4}\right),(K)$ hold and that $A \in C\left(\mathbb{R}^{N}, \mathbb{R}^{N}\right)$. Then there exists $\varepsilon_{0}>0$ such that for any $\varepsilon \in\left(0, \varepsilon_{0}\right)$, problem (1.1) admits a nontrivial mountain pass solution $u_{\varepsilon} \in X_{\varepsilon}$ such that $\left\|u_{\varepsilon}\right\|_{X_{\varepsilon}} \rightarrow 0$ as $\varepsilon \rightarrow 0$.

\section{Remark 1.1}

(i) unlike solutions with concentration phenomena constructed in some earlier works without the magnetic field, our nontrivial solutions are closed to the trivial solution.

(ii) If $A=0$ and $\alpha \nearrow 1$, then Theorem 1.1 reduces to a result of Ding and Lin in [15]. To our best knowledge, it seems that there is no result on the existence of solutions for singularly perturbed fractional Schrödinger equations with an external magnetic field.

(iii) In [32] it was proved that, in the singular limit for $\alpha \nearrow 1$, the operator $(1-\alpha) \varepsilon^{2 \alpha}(-\Delta)_{A_{\varepsilon}}^{\alpha}$ converges, in a suitable sense, to the classical local magnetic operator (1.4). Whence, up to multiplication by $1-\alpha$ the nonlocal theory is somehow consistent with the classical one.

The paper is organized as follows. In Section 2, we recall some necessary definitions and properties of the functional spaces. In Section 3, we provide some preliminary results. In Section 4 we prove Theorem 1.1. In Section 5, we get some results for problem (1.1) in the case $A$.

\section{Functional Setting}

For the convenience of the reader, in this part we recall some definitions and basic properties of fractional magnetic Sobolev spaces $H_{A_{\varepsilon}}^{\alpha}\left(\mathbb{R}^{N}, \mathbb{C}\right)$. For a wider treatment on these spaces, we 
refer the reader to [13]. Let $L^{2}\left(\mathbb{R}^{N}, \mathbb{C}\right)$ be the Lebesgue space of complex-valued functions with summable square, endowed with the real scalar product

$$
\langle u, v\rangle_{L^{2}}:=\operatorname{Re} \int_{\mathbb{R}^{N}} u \bar{v} d x
$$

for any $u, v \in L^{2}\left(\mathbb{R}^{N}, \mathbb{C}\right)$. For any $\alpha \in(0,1)$, the space $H_{A_{\varepsilon}}^{\alpha}\left(\mathbb{R}^{N}, \mathbb{C}\right)$ is defined by

$$
H_{A_{\varepsilon}}^{\alpha}\left(\mathbb{R}^{N}, \mathbb{C}\right)=\left\{u \in L^{2}\left(\mathbb{R}^{N}, \mathbb{C}\right):[u]_{\alpha, A_{\varepsilon}}<\infty\right\},
$$

where $[u]_{\alpha, A_{\varepsilon}}$ denotes the so-called magnetic Gagliardo semi-norm, that is

$$
[u]_{\alpha, A_{\varepsilon}}:=\left(\int_{\mathbb{R}^{2 N}} \frac{\left|u(x)-e^{\mathrm{i}(x-y) \cdot A_{\varepsilon}\left(\frac{x+y}{2}\right)} u(y)\right|^{2}}{|x-y|^{N+2 \alpha}} d x d y\right)^{\frac{1}{2}}
$$

and $H_{A_{\varepsilon}}^{\alpha}\left(\mathbb{R}^{N}, \mathbb{C}\right)$ is endowed with the norm

$$
\|u\|_{\alpha, A_{\varepsilon}}=\left([u]_{\alpha, A_{\varepsilon}}^{2}+\|u\|_{L^{2}}^{2}\right)^{1 / 2} .
$$

If $A=0$, then $H_{A_{\varepsilon}}^{\alpha}\left(\mathbb{R}^{N}, \mathbb{C}\right)$ reduces to the well-known fractional space $H^{\alpha}\left(\mathbb{R}^{N}\right)$. Also, $H_{A_{\varepsilon}}^{\alpha}\left(\mathbb{R}^{N}, \mathbb{C}\right)$ is a Hilbert space with the real scalar product

$$
\langle u, v\rangle_{\alpha, A_{\varepsilon}}:=\langle u, v\rangle_{L^{2}}+\operatorname{Re} \int_{\mathbb{R}^{2 N}} \frac{\left(u(x)-e^{\mathrm{i}(x-y) \cdot A_{\varepsilon}\left(\frac{x+y}{2}\right)} u(y)\right) \overline{\left(v(x)-e^{\mathrm{i}(x-y) \cdot A_{\varepsilon}\left(\frac{x+y}{2}\right)} v(y)\right)}}{|x-y|^{N+2 \alpha}} d x d y,
$$

for any $u, v \in H_{A_{\varepsilon}}^{\alpha}\left(\mathbb{R}^{N}, \mathbb{C}\right)$. The operator $(-\Delta)_{A_{\varepsilon}}^{\alpha}: H_{A_{\varepsilon}}^{\alpha}\left(\mathbb{R}^{N}, \mathbb{C}\right) \rightarrow H_{A_{\varepsilon}}^{-\alpha}\left(\mathbb{R}^{N}, \mathbb{C}\right)$ is defined by

$$
\left\langle(-\Delta)_{A_{\varepsilon}}^{\alpha} u, v\right\rangle:=\operatorname{Re} \int_{\mathbb{R}^{2 N}} \frac{\left(u(x)-e^{\mathrm{i}(x-y) \cdot A_{\varepsilon}\left(\frac{x+y}{2}\right)} u(y)\right) \overline{\left(v(x)-e^{\mathrm{i}(x-y) \cdot A_{\varepsilon}\left(\frac{x+y}{2}\right)} v(y)\right)}}{|x-y|^{N+2 \alpha}} d x d y,
$$

via duality. Furthermore, the space $D_{A_{\varepsilon}}^{\alpha}\left(\mathbb{R}^{N}, \mathbb{C}\right)$ is defined as

$$
D_{A_{\varepsilon}}^{\alpha}\left(\mathbb{R}^{N}, \mathbb{C}\right):=\left\{u \in L^{2_{\alpha}^{*}}\left(\mathbb{R}^{N}, \mathbb{C}\right):[u]_{\alpha, A_{\varepsilon}}<\infty\right\} .
$$

and endowed with the norm $[\cdot]_{\alpha, A_{\varepsilon}}$. We recall (cf. [13, Lemma 3.5]) the following embedding

Proposition 2.1 (Magnetic embeddings). The embeddings

$$
D_{A_{\epsilon}}^{\alpha}\left(\mathbb{R}^{N}, \mathbb{C}\right) \hookrightarrow L^{2_{\alpha}^{*}}\left(\mathbb{R}^{N}, \mathbb{C}\right), \quad H_{A_{\epsilon}}^{\alpha}\left(\mathbb{R}^{N}, \mathbb{C}\right) \hookrightarrow L^{\nu}\left(\mathbb{R}^{N}, \mathbb{C}\right),
$$

is continuous for any $\nu \in\left[2,2_{\alpha}^{*}\right]$. Moreover, the embedding

$$
H_{A_{\epsilon}}^{\alpha}\left(\mathbb{R}^{N}, \mathbb{C}\right) \hookrightarrow \hookrightarrow L_{\text {loc }}^{\nu}\left(\mathbb{R}^{N}, \mathbb{C}\right)
$$

is compact for any $\nu \in\left[1,2_{\alpha}^{*}\right)$.

In this paper, we will use the following subspace of $D_{A_{\varepsilon}}^{\alpha}\left(\mathbb{R}^{N}\right)$ defined by

$$
X_{\varepsilon}:=\left\{u \in D_{A_{\varepsilon}}^{\alpha}\left(\mathbb{R}^{N}, \mathbb{C}\right): \int_{\mathbb{R}^{N}} V(x)|u|^{2} d x<\infty\right\}
$$

with the norm

$$
\|u\|_{X_{\varepsilon}}=\left([u]_{\alpha, A_{\varepsilon}}^{2}+\int_{\mathbb{R}^{N}} V(x)|u|^{2} d x\right)^{1 / 2},
$$

where $V$ is nonnegative. For any $\varepsilon>0$, the norm $\|\cdot\|_{X_{\varepsilon}}$ is equivalent to the following norm

$$
\|u\|_{\varepsilon}:=\left([u]_{\alpha, A_{\varepsilon}}^{2}+\varepsilon^{-2 \alpha} \int_{\mathbb{R}^{N}} V(x)|u|^{2} d x\right)^{1 / 2}
$$

which will be used from time to time. 
Proposition 2.2 ( $X_{\varepsilon}$ embedding). If $\left(V_{2}\right)$ holds, the injection $X_{\varepsilon} \hookrightarrow H_{A_{\varepsilon}}^{\alpha}\left(\mathbb{R}^{N}, \mathbb{C}\right)$ is continuous.

Proof. Let $a>0$ be as in assumption $\left(V_{2}\right)$. For any $u \in X_{\varepsilon}$, we obtain

$$
\int_{\mathbb{R}^{N}} V(x)|u|^{2} d x=\int_{\mathbb{R}^{N} \backslash V^{a}} V(x)|u|^{2} d x+\int_{V^{a}} V(x)|u|^{2} d x .
$$

By the Hölder inequality,

$$
\int_{V^{a}}|u|^{2} d x \leq\left|V^{a}\right|^{1-\frac{2}{2_{\alpha}^{*}}}\left(\int_{V^{a}}|u|^{2_{\alpha}^{*}} d x\right)^{\frac{2}{2_{\alpha}^{*}}} \leq \frac{1}{S_{\alpha}^{\varepsilon}}\left|V^{a}\right|^{1-\frac{2}{2_{\alpha}^{*}}}[u]_{\alpha, A_{\varepsilon}}^{2},
$$

where $|\cdot|$ denotes the Lebesgue measure and $S_{\alpha}^{\varepsilon}$ is the best Sobolev constant of the magnetic Sobolev embedding $D_{A_{\varepsilon}}^{\alpha}\left(\mathbb{R}^{N}, \mathbb{C}\right) \hookrightarrow L^{2_{\alpha}^{*}}\left(\mathbb{R}^{N}, \mathbb{C}\right)$,

$$
S_{\alpha}^{\varepsilon}:=\inf _{u \in D_{A_{\varepsilon}}^{\alpha}\left(\mathbb{R}^{N}\right) \backslash\{0\}} \frac{[u]_{\alpha, A_{\varepsilon}}^{2}}{\|u\|_{L^{2 *}}^{2}} .
$$

Then, it follows from condition $\left(V_{2}\right)$ that

$$
\begin{aligned}
\|u\|_{X_{\varepsilon}}^{2} & \geq \frac{1}{2}[u]_{\alpha, A_{\varepsilon}}^{2}+\frac{1}{2}[u]_{\alpha, A_{\varepsilon}}^{2}+\int_{\mathbb{R}^{N} \backslash V^{a}} V(x)|u|^{2} d x \\
& \geq \frac{1}{2}[u]_{\alpha, A_{\varepsilon}}^{2}+\frac{1}{2} S_{\alpha}^{\varepsilon}\left|V^{a}\right|^{\frac{2}{2_{\alpha}^{*}}-1} \int_{V^{a}}|u|^{2} d x+a \int_{\mathbb{R}^{N} \backslash V^{a}}|u|^{2} d x \\
& \geq \min \left\{\frac{1}{4}, \frac{1}{4} S_{\alpha}^{\varepsilon}\left|V^{a}\right|^{\frac{2}{2_{\alpha}^{*}}-1}, \frac{a}{2}\right\}\|u\|_{\alpha, A_{\varepsilon}}^{2},
\end{aligned}
$$

which implies that $X_{\varepsilon}$ is continuously embedded in $H_{A_{\varepsilon}}^{\alpha}\left(\mathbb{R}^{N}, \mathbb{C}\right)$.

\section{Preliminary Results}

Throughout this section, we assume that conditions $\left(f_{1}\right)-\left(f_{4}\right),\left(V_{1}\right)-\left(V_{2}\right)$ and $(K)$ are satisfied. Without loss of generality, we assume that

$$
V(0)=\min _{x \in \mathbb{R}^{N}} V(x)=0 .
$$

To obtain the solution of problem (1.1), we will use the following equivalent form

$$
(-\Delta)_{A_{\varepsilon}}^{\alpha} u+\varepsilon^{-2 \alpha} V(x) u=\varepsilon^{-2 \alpha} f(x,|u|) u+\varepsilon^{-2 \alpha} K(x)|u|^{2_{\alpha}^{*}-2} u,
$$

where $\varepsilon>0$. The energy functional associated with (3.1) on $X_{\varepsilon}$ is defined as follows

$$
I_{\varepsilon}(u):=\frac{1}{2}[u]_{\alpha, A_{\varepsilon}}^{2}+\frac{\varepsilon^{-2 \alpha}}{2} \int_{\mathbb{R}^{N}} V(x)|u|^{2} d x-\varepsilon^{-2 \alpha} \int_{\mathbb{R}^{N}} F(x,|u|) d x-\frac{\varepsilon^{-2 \alpha}}{2_{\alpha}^{*}} \int_{\mathbb{R}^{N}} K(x)|u|^{2_{\alpha}^{*}} d x .
$$

It is easy to check that $I_{\varepsilon} \in C^{1}\left(X_{\varepsilon}, \mathbb{R}\right)$ and that any critical point for $I_{\varepsilon}$ is a weak solution of problem (3.1). In the following, let $\left\{u_{n}\right\}_{n \in \mathbb{N}}$ be a (PS) $)_{c}$ sequence for $I_{\varepsilon}$, namely $I_{\varepsilon}\left(u_{n}\right) \rightarrow c$ and $I_{\varepsilon}^{\prime}\left(u_{n}\right) \rightarrow 0$ in $X_{\varepsilon}^{*}$, as $n \rightarrow \infty$, where $X_{\varepsilon}^{*}$ is the dual space of $X_{\varepsilon}$.

By standard arguments, we get that $\left\{u_{n}\right\}_{n \in \mathbb{N}}$ is bounded in $X_{\varepsilon}$. Passing to a subsequence, still denoted by $\left\{u_{n}\right\}_{n \in \mathbb{N}}$, we assume that $u_{n} \rightarrow u$ weakly in $X_{\varepsilon}, u_{n} \rightarrow u$ in $L_{\text {loc }}^{2}\left(\mathbb{R}^{N}, \mathbb{C}\right), L_{\text {loc }}^{p}\left(\mathbb{R}^{N}, \mathbb{C}\right)$ and $u_{n}(x) \rightarrow u(x)$ a.e. in $\mathbb{R}^{N}$. It is easy to verify that $I_{\varepsilon}^{\prime}(u)=0$ and $I_{\varepsilon}(u) \geq 0$. Due to the loss of compactness for the critical embedding, we do not expect that the energy functional $I_{\varepsilon}$ satisfies the Palais-Smale condition ((PS) condition for short) at any positive energy level, which makes the study via variational methods rather complicated. As in the celebrated contribution by Brézis and Nirenberg [3], we show that the (PS) condition holds for energy level less than some positive constant. Then, by the Minimax Theorem, we get the existence of solutions to (3.1). 
First of all, we give some preliminary results to show that $I_{\varepsilon}$ satisfies the $(\mathrm{PS})_{\mathrm{c}}$ at energy levels $c$ below some constant. From now on $\left\{u_{n}\right\}_{n \in \mathbb{N}}$ denotes the aforementioned (PS) sequence.

Lemma 3.1 (Vanishing). There is a subsequence $\left\{u_{n_{j}}\right\}_{j \in \mathbb{N}}$ of the (PS)c sequence $\left\{u_{n}\right\}_{n \in \mathbb{N}} \subset X_{\varepsilon}$ such that for any $\sigma>0$, there exists $r_{\sigma}>0$, which satisfies

$$
\limsup _{j \rightarrow \infty} \int_{B_{j} \backslash B_{r}}\left|u_{n_{j}}\right|^{s} d x \leq \sigma
$$

for any $r \geq r_{\sigma}$, where $s=2$ or $s=p$, and $B_{r}=\left\{x \in \mathbb{R}^{N}:|x|<r\right\}$.

Proof. For any $r>0, \int_{B_{r}}\left|u_{n}\right|^{s} d x \rightarrow \int_{B_{r}}|u|^{s} d x$ as $n \rightarrow \infty$. Then, there exists $n_{j} \in \mathbb{N}$ with $n_{j+1}>n_{j}$ such that

$$
\int_{B_{j}}\left|u_{n_{j}}\right|^{s} d x-\int_{B_{j}}|u|^{s} d x<\frac{1}{j} .
$$

For any $\sigma>0$, there exists $r_{\sigma}>0$ such that for any $r \geq r_{\sigma}$,

$$
\int_{\mathbb{R}^{N} \backslash B_{r}}|u|^{s} d x<\sigma .
$$

If $j>r_{\sigma}$, we have

$$
\begin{aligned}
\int_{B_{j} \backslash B_{r}}\left|u_{n_{j}}\right|^{s} d x & =\int_{B_{j}}\left|u_{n_{j}}\right|^{s} d x-\int_{B_{j}}|u|^{s} d x+\int_{B_{j} \backslash B_{r}}|u|^{s} d x+\int_{B_{r}}|u|^{s} d x-\int_{B_{r}}\left|u_{n_{j}}\right|^{s} d x \\
& <\frac{1}{j}+\sigma+\int_{B_{r}}|u|^{s} d x-\int_{B_{r}}\left|u_{n_{j}}\right|^{s} d x,
\end{aligned}
$$

for any $r \geq r_{\sigma}$, which yields the desired assertion.

Take $\varphi \in C_{0}^{\infty}\left(\mathbb{R}^{N}\right)$ such that $0 \leq \varphi \leq 1, \varphi(x)=1$ for $|x| \leq 1$ and $\varphi(x)=0$ for $|x| \geq 2$. Define

$$
\widehat{u}_{j}(x):=\varphi_{j}(x) u(x), \quad \varphi_{j}(x):=\varphi\left(\frac{2 x}{j}\right), \quad j \in \mathbb{N} .
$$

Then we have the following preliminary result.

Lemma 3.2 (Stability of truncation). For any $\varepsilon>0,\left\|\widehat{u}_{j}-u\right\|_{\varepsilon} \rightarrow 0$ as $j \rightarrow \infty$.

Proof. It is readily seen that

$$
\begin{aligned}
{\left[\widehat{u}_{j}-u\right]_{\alpha, A_{\varepsilon}} \leq } & 2 \int_{\mathbb{R}^{2 N}} \frac{u^{2}(x)\left(\varphi_{j}(x)-\varphi_{j}(y)\right)^{2}}{|x-y|^{N+2 \alpha}} d x d y \\
& +2 \int_{\mathbb{R}^{2 N}} \frac{\left(\varphi_{j}(y)-1\right)^{2}\left|u(x)-e^{\mathrm{i}(x-y) \cdot A_{\varepsilon}\left(\frac{x+y}{2}\right)} u(y)\right|^{2}}{|x-y|^{N+2 \alpha}} d x d y .
\end{aligned}
$$

Note that $u \in X_{\varepsilon},\left|\varphi_{j}(y)-1\right| \leq 2$ and $\varphi_{j}(y)-1 \rightarrow 0$ a.e. as $j \rightarrow \infty$. Then, the Dominated Convergence Theorem yields

$$
\int_{\mathbb{R}^{2 N}} \frac{\left(\varphi_{j}(y)-1\right)^{2}\left|u(x)-e^{\mathrm{i}(x-y) \cdot A_{\varepsilon}\left(\frac{x+y}{2}\right)} u(y)\right|^{2}}{|x-y|^{N+2 \alpha}} d x d y \rightarrow 0
$$

as $j \rightarrow \infty$. In the following, we will prove that

$$
\int_{\mathbb{R}^{2 N}} \frac{u^{2}(x)\left(\varphi_{j}(x)-\varphi_{j}(y)\right)^{2}}{|x-y|^{N+2 \alpha}} d x d y \rightarrow 0 \quad \text { as } j \rightarrow \infty .
$$

Note that

$$
\begin{aligned}
\mathbb{R}^{N} \times \mathbb{R}^{N} & =\left(\left(\mathbb{R}^{N} \backslash B_{j}\right) \cup B_{j}\right) \times\left(\left(\mathbb{R}^{N} \backslash B_{j}\right) \cup B_{j}\right) \\
& =\left(\left(\mathbb{R}^{N} \backslash B_{j}\right) \times\left(\mathbb{R}^{N} \backslash B_{j}\right)\right) \cup\left(B_{j} \times \mathbb{R}^{N}\right) \cup\left(\left(\mathbb{R}^{N} \backslash B_{j}\right) \times B_{j}\right) .
\end{aligned}
$$


(i) $(x, y) \in\left(\mathbb{R}^{N} \backslash B_{j}\right) \times\left(\mathbb{R}^{N} \backslash B_{j}\right)$, we have $\varphi_{j}(x)=\varphi_{j}(y)=0$.

(ii) $(x, y) \in B_{j} \times \mathbb{R}^{N}$. One has

$$
\begin{aligned}
& \int_{B_{j}} d x \int_{\left\{y \in \mathbb{R}^{N}:|x-y| \leq \frac{1}{2} j\right\}} \frac{u^{2}(x)\left|\varphi_{j}(x)-\varphi_{j}(y)\right|^{2}}{|x-y|^{N+2 \alpha}} d y \\
& =\int_{B_{j}} d x \int_{\left\{y \in \mathbb{R}^{N}:|x-y| \leq \frac{1}{2} j\right\}} \frac{u^{2}(x)|\nabla \varphi(\xi)|^{2}\left|\frac{2(x-y)}{j}\right|^{2}}{|x-y|^{N+2 \alpha}} d y \\
& \leq C j^{-2} \int_{B_{j}} d x \int_{\left\{y \in \mathbb{R}^{N}:|x-y| \leq \frac{1}{2} j\right\}} \frac{u^{2}(x)}{|x-y|^{N+2 \alpha-2}} d y \\
& =C j^{-2 \alpha} \int_{B_{j}} u^{2}(x) d x,
\end{aligned}
$$

where $\xi=\frac{2 y}{j}+\tau \frac{2(x-y)}{j}, \tau \in(0,1)$ and

$$
\begin{aligned}
& \int_{B_{j}} d x \int_{\left\{y \in \mathbb{R}^{N}:|x-y|>\frac{1}{2} j\right\}} \frac{u^{2}(x)\left|\varphi_{j}(x)-\varphi_{j}(y)\right|^{2}}{|x-y|^{N+2 \alpha}} d y \\
& \leq C \int_{B_{j}} d x \int_{\left\{y \in \mathbb{R}^{N}:|x-y|>\frac{1}{2} j\right\}} \frac{u^{2}(x)}{|x-y|^{N+2 \alpha}} d y \\
& =C j^{-2 \alpha} \int_{B_{j}} u^{2}(x) d x .
\end{aligned}
$$

(iii) $(x, y) \in\left(\mathbb{R}^{N} \backslash B_{j}\right) \times B_{j}$. If $|x-y| \leq \frac{1}{2} j$, then $|x| \leq|x-y|+|y| \leq \frac{3}{2} j$. Furthermore,

$$
\begin{aligned}
& \int_{\mathbb{R}^{N} \backslash B_{j}} d x \int_{\left\{y \in B_{j}:|x-y| \leq \frac{1}{2} j\right\}} \frac{u^{2}(x)\left|\varphi_{j}(x)-\varphi_{j}(y)\right|^{2}}{|x-y|^{N+2 \alpha}} d y \\
& \leq C j^{-2} \int_{B_{\frac{3}{2} j}} d x \int_{\left\{y \in B_{j}:|x-y| \leq \frac{1}{2} j\right\}} \frac{u^{2}(x)}{|x-y|^{N+2 \alpha-2}} d y \\
& \leq C j^{-2 \alpha} \int_{B_{\frac{3}{2} j}} u^{2}(x) d x .
\end{aligned}
$$

Notice that, for any $k>4$, there holds

$$
\mathbb{R}^{N} \backslash B_{j} \subset B_{\frac{k}{2} j} \cup\left(\mathbb{R}^{N} \backslash B_{\frac{k}{2} j}\right) .
$$

If $|x-y|>\frac{1}{2} j$, then we obtain

$$
\begin{aligned}
& \int_{B_{\frac{k}{2} j}} d x \int_{\left\{y \in B_{j}:|x-y|>\frac{1}{2} j\right\}} \frac{u^{2}(x)\left|\varphi_{j}(x)-\varphi_{j}(y)\right|^{2}}{|x-y|^{N+2 \alpha}} d y \\
& \leq C \int_{B_{\frac{k}{2} j}} d x \int_{\left\{y \in B_{j}:|x-y|>\frac{1}{2} j\right\}} \frac{u^{2}(x)}{|x-y|^{N+2 \alpha}} d y \\
& \leq C j^{-2 \alpha} \int_{B_{\frac{k}{2} j}} u^{2}(x) d x .
\end{aligned}
$$


If $(x, y) \in\left(\mathbb{R}^{N} \backslash B_{\frac{k}{2} j}\right) \times B_{j}$, then $|x-y| \geq|x|-|y| \geq \frac{|x|}{2}+\frac{k}{4} j-j>\frac{|x|}{2}$. Hölder inequality yields

$$
\begin{aligned}
& \int_{\mathbb{R}^{N} \backslash B_{\frac{k}{2} j}} d x \int_{\left\{y \in B_{j}:|x-y|>\frac{1}{2} j\right\}} \frac{u^{2}(x)\left|\varphi_{j}(x)-\varphi_{j}(y)\right|^{2}}{|x-y|^{N+2 \alpha}} d y \\
& \leq C \int_{\mathbb{R}^{N} \backslash B_{\frac{k}{2} j}} d x \int_{\left\{y \in B_{j}:|x-y|>\frac{1}{2} j\right\}} \frac{u^{2}(x)}{|x|^{N+2 \alpha}} d y \\
& \leq C j^{N} \int_{\mathbb{R}^{N} \backslash B_{\frac{k}{2} j}} \frac{u^{2}(x)}{|x|^{N+2 \alpha}} d x \\
& \leq C k^{-N}\left(\int_{\mathbb{R}^{N} \backslash B_{\frac{k}{2} j}}|u(x)|^{2_{\alpha}^{*}} d x\right)^{\frac{2}{2_{\alpha}^{*}}} .
\end{aligned}
$$

By combining (i), (ii) and (iii), we get

$$
\begin{aligned}
& \int_{\mathbb{R}^{2 N}} \frac{u^{2}(x)\left|\varphi_{j}(x)-\varphi_{j}(y)\right|^{2}}{|x-y|^{N+2 \alpha}} d x d y \\
& =\left(\int_{B_{j} \times \mathbb{R}^{N}}+\int_{\left(\mathbb{R}^{N} \backslash B_{j}\right) \times B_{j}}\right) \frac{u^{2}(x)\left|\varphi_{j}(x)-\varphi_{j}(y)\right|^{2}}{|x-y|^{N+2 \alpha}} d x d y \\
& \leq C j^{-2 \alpha} \int_{B_{\frac{k}{2} j}} u^{2}(x) d x+C k^{-N}\left(\int_{\mathbb{R}^{N} \backslash B_{\frac{k}{2} j}}|u(x)|^{2_{\alpha}^{*}} d x\right)^{2 / 2_{\alpha}^{*}} \\
& \leq C j^{-2 \alpha}+C k^{-N} .
\end{aligned}
$$

Therefore, we have

$$
\begin{aligned}
& \limsup _{j \rightarrow \infty} \int_{\mathbb{R}^{2 N}} \frac{u^{2}(x)\left(\varphi_{j}(x)-\varphi_{j}(y)\right)^{2}}{|x-y|^{N+2 \alpha}} d x d y \\
& =\lim _{k \rightarrow \infty} \limsup _{j \rightarrow \infty} \int_{\mathbb{R}^{2 N}} \frac{u^{2}(x)\left(\varphi_{j}(x)-\varphi_{j}(y)\right)^{2}}{|x-y|^{N+2 \alpha}} d x d y=0 .
\end{aligned}
$$

It follows from (3.3) and (3.4) that

$$
\left[\widehat{u}_{j}-u\right]_{\alpha, A_{\varepsilon}} \rightarrow 0, \text { as } j \rightarrow \infty .
$$

Note that, as $j \rightarrow \infty$,

$$
\int_{\mathbb{R}^{N}} V(x)\left|\widehat{u}_{j}(x)-u(x)\right|^{2} d x=\int_{\mathbb{R}^{N}} V(x)\left(\varphi_{j}(x)-1\right)^{2} u^{2}(x) d x \rightarrow 0,
$$

we deduce from the Dominated Convergence Theorem that

$$
\int_{\mathbb{R}^{N}} V(x)\left|\widehat{u}_{j}(x)-u(x)\right|^{2} d x \rightarrow 0
$$

as $j \rightarrow \infty$. Thus $\left\|\widehat{u}_{j}-u\right\|_{\varepsilon} \rightarrow 0$ as $j \rightarrow \infty$.

Lemma 3.3 (Shifted Palais-Smale). Let $\left\{u_{n_{j}}\right\}_{j \in \mathbb{N}} \subset X_{\varepsilon}$ be the sequence introduced in Lemma 3.1. Moreover, for any $j \in \mathbb{N}$, let us denote

$$
u_{n_{j}}^{1}:=u_{n_{j}}-\widehat{u}_{j}, \quad j \geq 1 .
$$

Then, $I_{\varepsilon}\left(u_{n_{j}}^{1}\right) \rightarrow c-I_{\varepsilon}(u)$ and $I_{\varepsilon}^{\prime}\left(u_{n_{j}}^{1}\right) \rightarrow 0$ in $X_{\varepsilon}^{*}$ as $j \rightarrow \infty$. 
Proof. Notice that, it holds

$$
\begin{aligned}
& \quad I_{\varepsilon}\left(u_{n_{j}}^{1}\right)-I_{\varepsilon}\left(u_{n_{j}}\right)+I_{\varepsilon}\left(\widehat{u}_{j}\right) \\
& =\int_{\mathbb{R}^{2 N}} \frac{\left|\widehat{u}_{j}(x)-e^{\mathrm{i}(x-y) \cdot A_{\varepsilon}\left(\frac{x+y}{2}\right)} \widehat{u}_{j}(y)\right|^{2}}{|x-y|^{N+2 \alpha}} d x d y \\
& \quad-\operatorname{Re} \int_{\mathbb{R}^{2 N}} \frac{\left(u_{n_{j}}(x)-e^{\mathrm{i}(x-y) \cdot A_{\varepsilon}\left(\frac{x+y}{2}\right)} u_{n_{j}}(y)\right) \overline{\left(\widehat{u}_{j}(x)-e^{\left.\mathrm{i}(x-y) \cdot A_{\varepsilon}\left(\frac{x+y}{2}\right) \widehat{u}_{j}(y)\right)}\right.}}{|x-y|^{N+2 \alpha}} d x d y \\
& \quad+\varepsilon^{-2 \alpha} \int_{\mathbb{R}^{N}} V(x)\left|\widehat{u}_{j}\right|^{2} d x-\varepsilon^{-2 \alpha} \operatorname{Re} \int_{\mathbb{R}^{N}} V(x) u_{n_{j}} \widehat{\widehat{u}}_{j} d x \\
& \quad+\varepsilon^{-2 \alpha} \int_{\mathbb{R}^{N}}\left(F\left(x,\left|u_{n_{j}}\right|\right)-F\left(x,\left|u_{n_{j}}-\widehat{u}_{j}\right|\right)-F\left(x,\left|\widehat{u}_{j}\right|\right)\right) d x \\
& \quad+\frac{\varepsilon^{-2 \alpha}}{2_{\alpha}^{*}} \int_{\mathbb{R}^{N}} K(x)\left(\left|u_{n_{j}}\right|^{2_{\alpha}^{*}}-\left|u_{n_{j}}-\widehat{u}_{j}\right|^{2_{\alpha}^{*}}-\left|\widehat{u}_{j}\right|^{2_{\alpha}^{*}}\right) d x .
\end{aligned}
$$

As $u_{n_{j}} \rightarrow u$ weakly in $X_{\varepsilon}$ and $\widehat{u}_{j} \rightarrow u$ strongly in $X_{\varepsilon}$, we could derive that

$$
\begin{aligned}
\int_{\mathbb{R}^{2 N}} & \frac{\left|\widehat{u}_{j}(x)-e^{\mathrm{i}(x-y) \cdot A_{\varepsilon}\left(\frac{x+y}{2}\right)} \widehat{u}_{j}(y)\right|^{2}}{|x-y|^{N+2 \alpha}} d x d y \\
& -\operatorname{Re} \int_{\mathbb{R}^{2 N}} \frac{\left(u_{n_{j}}(x)-e^{\mathrm{i}(x-y) \cdot A_{\varepsilon}\left(\frac{x+y}{2}\right)} u_{n_{j}}(y)\right) \overline{\left(\widehat{u}_{j}(x)-e^{\mathrm{i}(x-y) \cdot A_{\varepsilon}\left(\frac{x+y}{2}\right)} \widehat{u}_{j}(y)\right)}}{|x-y|^{N+2 \alpha}} d x d y \rightarrow 0
\end{aligned}
$$

and, as $j \rightarrow \infty$,

$$
\int_{\mathbb{R}^{N}} V(x)\left|\widehat{u}_{j}\right|^{2} d x-\operatorname{Re} \int_{\mathbb{R}^{N}} V(x) u_{n_{j}} \overline{\widehat{u}_{j}} d x \rightarrow 0 .
$$

Arguing as for the proof of the Brézis-Lieb Lemma and recalling that $\widehat{u}_{j} \rightarrow u$ strongly in $X_{\varepsilon}$ as $j \rightarrow \infty$, it is easy to prove that

$$
\begin{gathered}
\int_{\mathbb{R}^{N}}\left(F\left(x, u_{n_{j}}\right)-F\left(x, u_{n_{j}}-\widehat{u}_{j}\right)-F\left(x, \widehat{u}_{j}\right)\right) d x \rightarrow 0, \\
\int_{\mathbb{R}^{N}} K(x)\left(\left|u_{n_{j}}\right|^{2_{\alpha}^{*}}-\left|u_{n_{j}}-\widehat{u}_{j}\right|^{2_{\alpha}^{*}}-\left|\widehat{u}_{j}\right|^{2_{\alpha}^{*}}\right) d x \rightarrow 0 .
\end{gathered}
$$

Thus, $I_{\varepsilon}\left(u_{n_{j}}^{1}\right) \rightarrow c-I_{\varepsilon}(u)$, as $j \rightarrow \infty$. Taking now $\phi \in X_{\varepsilon}$ with $\|\phi\|_{\varepsilon} \leq 1$, we obtain

$$
\begin{aligned}
& \left\langle I_{\varepsilon}^{\prime}\left(u_{n_{j}}^{1}\right)-I_{\varepsilon}^{\prime}\left(u_{n_{j}}\right)+I_{\varepsilon}^{\prime}\left(\widehat{u}_{j}\right), \phi\right\rangle \\
= & \varepsilon^{-2 \alpha} \operatorname{Re} \int_{\mathbb{R}^{N}}\left(f\left(x,\left|u_{n_{j}}\right|\right) u_{n_{j}}-f\left(x,\left|u_{n_{j}}-\widehat{u}_{j}\right|\right)\left(u_{n_{j}}-\widehat{u}_{j}\right)-f\left(x,\left|\widehat{u}_{j}\right|\right) \widehat{u}_{j}\right) \bar{\phi} d x \\
& +\varepsilon^{-2 \alpha} \operatorname{Re} \int_{\mathbb{R}^{N}} K(x)\left(\left|u_{n_{j}}\right|^{2_{\alpha}^{*}-2} u_{n_{j}}-\left|u_{n_{j}}-\widehat{u}_{j}\right|^{2_{\alpha}^{*}-2}\left(u_{n_{j}}-\widehat{u}_{j}\right)-\left|\widehat{u}_{j}\right|^{2}-2 \widehat{u}_{j}\right) \bar{\phi} d x .
\end{aligned}
$$

It follows, again by a standard argument, that

$$
\left|\int_{\mathbb{R}^{N}} K(x)\left(\left|u_{n_{j}}\right|^{2_{\alpha}^{*}-2} u_{n_{j}}-\left|u_{n_{j}}-\widehat{u}_{j}\right|^{2 *}-2\left(u_{n_{j}}-\widehat{u}_{j}\right)-\left|\widehat{u}_{j}\right|^{2}-2 \widehat{u}_{j}\right) \bar{\phi} d x\right| \rightarrow 0
$$


uniformly in $\phi \in X_{\varepsilon}$ with $\|\phi\|_{\varepsilon} \leq 1$, as $j \rightarrow \infty$. Meanwhile, we have

$$
\begin{aligned}
& \left|\int_{\mathbb{R}^{N}}\left(f\left(x,\left|u_{n_{j}}\right|\right) u_{n_{j}}-f\left(x,\left|u_{n_{j}}-\widehat{u}_{j}\right|\right)\left(u_{n_{j}}-\widehat{u}_{j}\right)-f\left(x,\left|\widehat{u}_{j}\right|\right) \widehat{u}_{j}\right) \bar{\phi} d x\right| \\
\leq & \int_{B_{r}}\left|f\left(x,\left|u_{n_{j}}\right|\right) u_{n_{j}}-f\left(x,\left|u_{n_{j}}-\widehat{u}_{j}\right|\right)\left(u_{n_{j}}-\widehat{u}_{j}\right)-f\left(x,\left|\widehat{u}_{j}\right|\right) \widehat{u}_{j}\right| \cdot|\phi| d x \\
& +\int_{\mathbb{R}^{N} \backslash B_{r}}\left|f\left(x,\left|u_{n_{j}}\right|\right) u_{n_{j}}-f\left(x,\left|u_{n_{j}}-\widehat{u}_{j}\right|\right)\left(u_{n_{j}}-\widehat{u}_{j}\right)-f\left(x,\left|\widehat{u}_{j}\right|\right) \widehat{u}_{j}\right| \cdot|\phi| d x
\end{aligned}
$$

for any $r \geq r_{\sigma}$, where $r_{\sigma}>0$ is as in Lemma 3.1. Since $\widehat{u}_{j} \rightarrow u$ and $u_{n_{j}} \rightarrow u$ in $L^{p}\left(B_{r}, \mathbb{C}\right)$, we get

$$
\int_{B_{r}}\left|f\left(x,\left|u_{n_{j}}\right|\right) u_{n_{j}}-f\left(x,\left|u_{n_{j}}-\widehat{u}_{j}\right|\right)\left(u_{n_{j}}-\widehat{u}_{j}\right)-f\left(x,\left|\widehat{u}_{j}\right|\right) \widehat{u}_{j}\right| \cdot|\phi| d x \rightarrow 0
$$

uniformly in $\phi \in X_{\varepsilon}$ with $\|\phi\|_{\varepsilon} \leq 1$. By $\left(f_{1}\right)$ and $\left(f_{2}\right)$, for any $t>0$ we obtain

$$
|f(x, t) t| \leq C\left(|t|+|t|^{p-1}\right),
$$

which implies (we recall that $\hat{u}_{j}=0$ on $\mathbb{R}^{N} \backslash B_{j}$ for any $j \geq 1$ )

$$
\begin{aligned}
& \int_{\mathbb{R}^{N} \backslash B_{r}}\left|f\left(x,\left|u_{n_{j}}\right|\right) u_{n_{j}}-f\left(x,\left|u_{n_{j}}-\widehat{u}_{j}\right|\right)\left(u_{n_{j}}-\widehat{u}_{j}\right)-f\left(x,\left|\widehat{u}_{j}\right|\right) \widehat{u}_{j}\right| \cdot|\phi| d x \\
= & \int_{B_{j} \backslash B_{r}}\left|f\left(x,\left|u_{n_{j}}\right|\right) u_{n_{j}}-f\left(x,\left|u_{n_{j}}-\widehat{u}_{j}\right|\right)\left(u_{n_{j}}-\widehat{u}_{j}\right)-f\left(x,\left|\widehat{u}_{j}\right|\right) \widehat{u}_{j}\right| \cdot|\phi| d x \\
\leq & C \int_{B_{j} \backslash B_{r}}\left(\left|u_{n_{j}}\right|+\left|\widehat{u}_{j}\right|+\left|u_{n_{j}}\right|^{p-1}+\left|\widehat{u}_{j}\right|^{p-1}\right) \cdot|\phi| d x .
\end{aligned}
$$

For any $\sigma>0$, by inequality (3.2), the Hölder inequality and Proposition 2.2, we have

$$
\begin{aligned}
& \limsup _{j \rightarrow \infty} \int_{B_{j} \backslash B_{r}}\left(\left|u_{n_{j}}\right|+\left|u_{n_{j}}\right|^{p-1}\right) \cdot|\phi| d x \\
\leq & \limsup _{j \rightarrow \infty}\left(\int_{B_{j} \backslash B_{r}}\left|u_{n_{j}}\right|^{2} d x\right)^{\frac{1}{2}}\left(\int_{B_{j} \backslash B_{r}}|\phi|^{2} d x\right)^{\frac{1}{2}} \\
+ & \limsup _{j \rightarrow \infty}\left(\int_{B_{j} \backslash B_{r}}\left|u_{n_{j}}\right|^{p} d x\right)^{\frac{p-1}{p}}\left(\int_{B_{j} \backslash B_{r}}|\phi|^{p} d x\right)^{\frac{1}{p}} \leq C\left(\sigma^{\frac{1}{2}}+\sigma^{\frac{p-1}{p}}\right) .
\end{aligned}
$$

Since $\widehat{u}_{j} \rightarrow u$ in $X_{\varepsilon}$ as $j \rightarrow \infty$, Proposition 2.2 yields that $\widehat{u}_{j} \rightarrow u$ in $L^{2}\left(\mathbb{R}^{N}, \mathbb{C}\right)$ and $L^{p}\left(\mathbb{R}^{N}, \mathbb{C}\right)$. Then, by the Hölder inequality, for any $r \geq r_{\sigma}$ (up to enlarging $r_{\sigma}$ ) we obtain

$$
\limsup _{j \rightarrow \infty} \int_{B_{j} \backslash B_{r}}\left(\left|\widehat{u}_{j}\right|+\left|\widehat{u}_{j}\right|^{p-1}\right) \cdot|\phi| d x=\int_{\mathbb{R}^{N} \backslash B_{r}}\left(|u|+|u|^{p-1}\right) \cdot|\phi| d x \leq C\left(\sigma^{\frac{1}{2}}+\sigma^{\frac{p-1}{p}}\right) .
$$

From (3.5)-(3.6), we have

$$
\limsup _{j \rightarrow \infty} \int_{\mathbb{R}^{N}}\left|f\left(x,\left|u_{n_{j}}\right|\right) u_{n_{j}}-f\left(x,\left|u_{n_{j}}-\widehat{u}_{j}\right|\right)\left(u_{n_{j}}-\widehat{u}_{j}\right)-f\left(x,\left|\widehat{u}_{j}\right|\right) \widehat{u}_{j}\right| \cdot|\phi| d x \leq C\left(\sigma^{\frac{1}{2}}+\sigma^{\frac{p-1}{p}}\right)
$$

uniformly in $\phi \in X_{\varepsilon}$ with $\|\phi\|_{\varepsilon} \leq 1$. Letting $\sigma \rightarrow 0$ yields,

$$
\limsup _{j \rightarrow \infty} \int_{\mathbb{R}^{N}}\left|f\left(x,\left|u_{n_{j}}\right|\right) u_{n_{j}}-f\left(x,\left|u_{n_{j}}-\widehat{u}_{j}\right|\right)\left(u_{n_{j}}-\widehat{u}_{j}\right)-f\left(x,\left|\widehat{u}_{j}\right|\right) \widehat{u}_{j}\right| \cdot|\phi| d x=0 .
$$

As $I_{\varepsilon}^{\prime}\left(u_{n_{j}}\right) \rightarrow 0$ and $I_{\varepsilon}^{\prime}\left(\widehat{u}_{j}\right) \rightarrow I_{\varepsilon}^{\prime}(u)=0$, we get that $I_{\varepsilon}^{\prime}\left(u_{n_{j}}^{1}\right) \rightarrow 0$, as $j \rightarrow \infty$. 
In what follows, we will show that for any $\varepsilon>0, I_{\varepsilon}$ satisfies $(\mathrm{PS})_{\mathrm{c}}$ condition for energy level $c$ below some positive constant depending on $\varepsilon$.

Lemma 3.4 (Palais-Smale). Let $K_{0}, K_{1}>0$ and $\mu>2$ be as in conditions $(K)$ and $\left(f_{3}\right)$ and let us denote by $c_{2}>0$ a suitable constant depending upon $f$. Then, for any $\varepsilon>0$, if

$$
-\infty<c<C_{0}(\varepsilon) \varepsilon^{N-2 \alpha}, \quad C_{0}(\varepsilon):=\left(\frac{S_{\alpha}^{\varepsilon}}{c_{2}+K_{1}}\right)^{\frac{2_{\alpha}^{*}}{2_{\alpha}^{*}-2}} \frac{\left(2_{\alpha}^{*}-\mu\right) K_{0}}{\mu 2_{\alpha}^{*}} .
$$

then $u_{n_{j}} \rightarrow u$ in $X_{\varepsilon}$ as $j \rightarrow \infty$.

Proof. By the definition of $\left\{u_{n_{j}}^{1}\right\}_{j \in \mathbb{N}}$ and Lemma 3.2, it suffices to have $u_{n_{j}}^{1} \rightarrow 0$ in $X_{\varepsilon}$ as $j \rightarrow \infty$. By means of conditions $\left(f_{3}\right)$ and $(K)$, we have

$$
\begin{aligned}
& I_{\varepsilon}\left(u_{n_{j}}^{1}\right)-\frac{1}{\mu}\left\langle I_{\varepsilon}^{\prime}\left(u_{n_{j}}^{1}\right), u_{n_{j}}^{1}\right\rangle \\
= & \left(\frac{1}{2}-\frac{1}{\mu}\right)\left[u_{n_{j}}^{1}\right]_{\alpha, A_{\varepsilon}}^{2}+\left(\frac{1}{2}-\frac{1}{\mu}\right) \varepsilon^{-2 \alpha} \int_{\mathbb{R}^{N}} V(x)\left|u_{n_{j}}^{1}\right|^{2} d x \\
& +\varepsilon^{-2 \alpha} \int_{\mathbb{R}^{N}}\left(\frac{1}{\mu} f\left(x,\left|u_{n_{j}}^{1}\right|\right)\left|u_{n_{j}}^{1}\right|^{2}-F\left(x,\left|u_{n_{j}}^{1}\right|\right)\right) d x+\varepsilon^{-2 \alpha}\left(\frac{1}{\mu}-\frac{1}{2_{\alpha}^{*}}\right) \int_{\mathbb{R}^{N}} K(x)\left|u_{n_{j}}^{1}\right|^{2_{\alpha}^{*}} d x \\
\geq & \varepsilon^{-2 \alpha}\left(\frac{1}{\mu}-\frac{1}{2_{\alpha}^{*}}\right) K_{0} \int_{\mathbb{R}^{N}}\left|u_{n_{j}}^{1}\right|^{2_{\alpha}^{*}} d x .
\end{aligned}
$$

Then, from Lemma 3.3, we get

$$
\limsup _{j \rightarrow \infty} \int_{\mathbb{R}^{N}}\left|u_{n_{j}}^{1}\right|^{2_{\alpha}^{*}} d x \leq \frac{\mu 2_{\alpha}^{*} \varepsilon^{2 \alpha}}{K_{0}\left(2_{\alpha}^{*}-\mu\right)}\left(c-I_{\varepsilon}(u)\right) .
$$

Suppose that $u_{n_{j}}^{1} \nrightarrow \rightarrow 0$ in $L^{2_{\alpha}^{*}}\left(\mathbb{R}^{N}, \mathbb{C}\right)$. Then, we have

$$
\liminf _{j \rightarrow \infty}\left\|u_{n_{j}}^{1}\right\|_{L^{2 *}}>0 .
$$

Noting that $\left\langle I_{\varepsilon}^{\prime}\left(u_{n_{j}}^{1}\right), u_{n_{j}}^{1}\right\rangle \rightarrow 0$ as $j \rightarrow \infty$, we have

$$
\begin{aligned}
& {\left[u_{n_{j}}^{1}\right]_{\alpha, A_{\varepsilon}}^{2}+\varepsilon^{-2 \alpha} \int_{\mathbb{R}^{N}} V(x)\left|u_{n_{j}}^{1}\right|^{2} d x } \\
= & \varepsilon^{-2 \alpha} \int_{\mathbb{R}^{N}} f\left(x,\left|u_{n_{j}}^{1}\right|\right)\left|u_{n_{j}}^{1}\right|^{2} d x+\varepsilon^{-2 \alpha} \int_{\mathbb{R}^{N}} K(x)\left|u_{n_{j}}^{1}\right|^{2_{\alpha}^{*}} d x+o_{j}(1) .
\end{aligned}
$$

It follows from (2.1) that

$$
\begin{aligned}
& S_{\alpha}^{\varepsilon}\left(\int_{\mathbb{R}^{N}}\left|u_{n_{j}}^{1}\right|^{2_{\alpha}^{*}} d x\right)^{\frac{2}{2_{\alpha}^{*}}} \leq\left[u_{n_{j}}^{1}\right]_{\alpha, A_{\varepsilon}}^{2} \\
= & \varepsilon^{-2 \alpha} \int_{\mathbb{R}^{N}} f\left(x,\left|u_{n_{j}}^{1}\right|\right)\left|u_{n_{j}}^{1}\right|^{2} d x+\varepsilon^{-2 \alpha} \int_{\mathbb{R}^{N}} K(x)\left|u_{n_{j}}^{1}\right|^{2_{\alpha}^{*}} d x-\varepsilon^{-2 \alpha} \int_{\mathbb{R}^{N}} V(x)\left|u_{n_{j}}^{1}\right|^{2} d x+o_{j}(1) .
\end{aligned}
$$

By $\left(f_{1}\right)$ and $\left(f_{2}\right)$, for any $\lambda>0$, there exists $C(\lambda)>0$ such that

$$
|f(x, t)| \leq \lambda+C(\lambda)|t|^{2_{\alpha}^{*}-2} .
$$


Thus

$$
\begin{aligned}
S_{\alpha}^{\varepsilon}\left(\int_{\mathbb{R}^{N}}\left|u_{n_{j}}^{1}\right|^{2_{\alpha}^{*}} d x\right)^{\frac{2}{2 *}} \leq & \lambda \varepsilon^{-2 \alpha} \int_{\mathbb{R}^{N}}\left|u_{n_{j}}^{1}\right|^{2} d x+C(\lambda) \varepsilon^{-2 \alpha} \int_{\mathbb{R}^{N}}\left|u_{n_{j}}^{1}\right|^{2_{\alpha}^{*}} d x \\
& +\varepsilon^{-2 \alpha} K_{1} \int_{\mathbb{R}^{N}}\left|u_{n_{j}}^{1}\right|^{2_{\alpha}^{*}} d x-\varepsilon^{-2 \alpha} \int_{\mathbb{R}^{N}} a\left|u_{n_{j}}^{1}\right|^{2} d x \\
& +\varepsilon^{-2 \alpha} \int_{\mathbb{R}^{N}} a\left|u_{n_{j}}^{1}\right|^{2} d x-\varepsilon^{-2 \alpha} \int_{\mathbb{R}^{N}} V(x)\left|u_{n_{j}}^{1}\right|^{2} d x+o_{j}(1) .
\end{aligned}
$$

Since $V^{a}$ has finite Lebesgue measure, we obtain $\left|V^{a} \backslash B_{R}\right| \rightarrow 0$ for $R \rightarrow \infty$. Then, for any $\eta>0$, there exists $R_{0}>0$ such that $\left|V^{a} \backslash B_{R}\right|<\eta$ for any $R \geq R_{0}$. We have

$$
\begin{aligned}
\int_{\mathbb{R}^{N}}(a-V(x))\left|u_{n_{j}}^{1}\right|^{2} d x & \leq \int_{V^{a}}(a-V(x))\left|u_{n_{j}}^{1}\right|^{2} d x \\
& =\int_{V^{a} \backslash B_{R_{0}}}(a-V(x))\left|u_{n_{j}}^{1}\right|^{2} d x+\int_{V^{a} \cap B_{R_{0}}}(a-V(x))\left|u_{n_{j}}^{1}\right|^{2} d x .
\end{aligned}
$$

Now the Hölder inequality gives

$$
\begin{aligned}
\int_{V^{a} \backslash B_{R_{0}}}(a-V(x))\left|u_{n_{j}}^{1}\right|^{2} d x & \leq \int_{V^{a} \backslash B_{R_{0}}} a\left|u_{n_{j}}^{1}\right|^{2} d x \\
& \leq a\left\|u_{n_{j}}^{1}\right\|_{L^{2_{\alpha}^{*}}}^{2}\left|V^{a} \backslash B_{R_{0}}\right|^{1-\frac{2}{2_{\alpha}^{*}}} \leq C \eta^{1-\frac{2}{2_{\alpha}^{*}}} .
\end{aligned}
$$

As $u_{n_{j}}^{1} \rightarrow 0$ weakly in $X, u_{n_{j}}^{1} \rightarrow 0$ in $L^{2}\left(B_{R_{0}}, \mathbb{C}\right)$, as $j \rightarrow \infty$. Then, for the above $\eta>0$, there exists $j_{0} \in \mathbb{N}$ such that for any $j \geq j_{0}$,

$$
\int_{V^{a} \cap B_{R_{0}}}(a-V(x))\left|u_{n_{j}}^{1}\right|^{2} d x \leq a \int_{B_{R_{0}}}\left|u_{n_{j}}^{1}\right|^{2} d x \leq a \eta .
$$

Let $\lambda=a / 2$. In terms of (3.11)-(3.12), there exists $c_{2}>0$ depending on $f$ such that

$$
S_{\alpha}^{\varepsilon}\left(\int_{\mathbb{R}^{N}}\left|u_{n_{j}}^{1}\right|^{2_{\alpha}^{*}} d x\right)^{\frac{2}{2_{\alpha}^{*}}} \leq\left(c_{2}+K_{1}\right) \varepsilon^{-2 \alpha} \int_{\mathbb{R}^{N}}\left|u_{n_{j}}^{1}\right|^{2_{\alpha}^{*}} d x+C \varepsilon^{-2 \alpha} \eta^{1-\frac{2}{2_{\alpha}^{*}}}+\varepsilon^{-2 \alpha} a \eta+o_{j}(1) .
$$

Letting $\eta \rightarrow 0$, we have

$$
S_{\alpha}^{\varepsilon}\left(\int_{\mathbb{R}^{N}}\left|u_{n_{j}}^{1}\right|^{2_{\alpha}^{*}} d x\right)^{\frac{2}{2_{\alpha}^{*}}} \leq\left(c_{2}+K_{1}\right) \varepsilon^{-2 \alpha} \int_{\mathbb{R}^{N}}\left|u_{n_{j}}^{1}\right|^{2_{\alpha}^{*}} d x+o_{j}(1) .
$$

From (3.7) and (3.8), we get

$$
S_{\alpha}^{\varepsilon} \leq\left(c_{2}+K_{1}\right) \varepsilon^{-2 \alpha}\left(\frac{\mu 2_{\alpha}^{*} \varepsilon^{2 \alpha}}{K_{0}\left(2_{\alpha}^{*}-\mu\right)}\left(c-I_{\varepsilon}(u)\right)\right)^{1-\frac{2}{2_{\alpha}^{*}}} .
$$

Then $C_{0}(\varepsilon) \varepsilon^{N-2 \alpha} \leq c-I_{\varepsilon}(u) \leq c$. If $c<C_{0}(\varepsilon) \varepsilon^{N-2 \alpha}$, we get a contradiction, which implies

$$
u_{n_{j}}^{1} \rightarrow 0 \quad \text { in } L^{2_{\alpha}^{*}}\left(\mathbb{R}^{N}, \mathbb{C}\right) \text {. }
$$

It follows from (3.10) that

$$
\left.\left|\int_{\mathbb{R}^{N}} f\left(x,\left|u_{n_{j}}^{1}\right|\right)\right| u_{n_{j}}^{1}\right|^{2} d x \mid \leq \int_{\mathbb{R}^{N}}\left(\lambda\left|u_{n_{j}}^{1}\right|^{2}+C(\lambda)\left|u_{n_{j}}^{1}\right|^{2_{\alpha}^{*}}\right) d x .
$$

As $\left\{u_{n_{j}}^{1}\right\}_{j \in \mathbb{N}}$ is bounded in $L^{2}\left(\mathbb{R}^{N}\right)$, we have

$$
\limsup _{j \rightarrow \infty} \int_{\mathbb{R}^{N}} f\left(x,\left|u_{n_{j}}^{1}\right|\right)\left|u_{n_{j}}^{1}\right|^{2} d x=\limsup _{\lambda \rightarrow 0} \limsup _{j \rightarrow \infty} \int_{\mathbb{R}^{N}} f\left(x,\left|u_{n_{j}}^{1}\right|\right)\left|u_{n_{j}}^{1}\right|^{2} d x=0 .
$$


By (3.9), it follows that $u_{n_{j}}^{1} \rightarrow 0$ in $X_{\varepsilon}$ as $j \rightarrow \infty$.

Next we provide a result to show that $I_{\varepsilon}$ has a Mountain Pass geometry.

Lemma 3.5 (Mountain Pass geometry I). For any $\varepsilon>0$ and $\delta>0$, there exist $t_{0}=t_{0}(\varepsilon, \delta)>0$ and $\psi_{\varepsilon, \delta} \in X_{\varepsilon}$ such that $I_{\varepsilon}\left(t_{0} \psi_{\varepsilon, \delta}\right)<0$.

Proof. We first verify that

$$
\inf \left\{\int_{\mathbb{R}^{N}} \frac{|\phi(x)-\phi(y)|^{2}}{|x-y|^{N+2 \alpha}} d x d y: \phi \in C_{0}^{\infty}\left(\mathbb{R}^{N}\right) \text { with }\|\phi\|_{L^{q}\left(\mathbb{R}^{N}\right)}=1\right\}=0 .
$$

Let $\phi \in C_{0}^{\infty}\left(\mathbb{R}^{N}\right)$ with $\|\phi\|_{L^{q}\left(\mathbb{R}^{N}\right)}=1$ and $\operatorname{supp} \phi \subset B_{r_{0}}$, where $r_{0}>0$. Then we have

$$
\int_{\mathbb{R}^{N}}\left|\delta^{\frac{N}{q}} \phi(\delta x)\right|^{q} d x=1
$$

and, as $\delta \rightarrow 0$,

$$
\int_{\mathbb{R}^{2 N}} \frac{\left|\delta^{\frac{N}{q}} \phi(\delta x)-\delta^{\frac{N}{q}} \phi(\delta y)\right|^{2}}{|x-y|^{N+2 \alpha}} d x d y=\delta^{\frac{2 N-(N-2 \alpha) q}{q}} \int_{\mathbb{R}^{2 N}} \frac{|\phi(x)-\phi(y)|^{2}}{|x-y|^{N+2 \alpha}} d x d y \rightarrow 0 .
$$

Hence, for any $\delta>0$, there exist $r_{\delta}>0$ and $\phi_{\delta} \in C_{0}^{\infty}\left(\mathbb{R}^{N}\right)$ with $\left\|\phi_{\delta}\right\|_{L^{q}\left(\mathbb{R}^{N}\right)}=1$ and $\operatorname{supp} \phi_{\delta} \subset B_{r_{\delta}}$ such that

$$
\int_{\mathbb{R}^{2 N}} \frac{\left|\phi_{\delta}(x)-\phi_{\delta}(y)\right|^{2}}{|x-y|^{N+2 \alpha}} d x d y \leq C \delta^{\frac{2 N-(N-2 \alpha) q}{q}} .
$$

Let $\psi_{\delta}(x):=e^{\mathrm{i} A(0) \cdot x} \phi_{\delta}(x)$ and $\psi_{\varepsilon, \delta}(x):=\psi_{\delta}\left(\varepsilon^{-1} x\right)$. By $\left(f_{4}\right)$, for any $t>0$ we get

$$
\begin{aligned}
I_{\varepsilon}\left(t \psi_{\varepsilon, \delta}\right) \leq & \frac{t^{2}}{2}\left[\psi_{\varepsilon, \delta}\right]_{\alpha, A_{\varepsilon}}^{2}+t^{2} \frac{\varepsilon^{-2 \alpha}}{2} \int_{\mathbb{R}^{N}} V(x)\left|\psi_{\varepsilon, \delta}(x)\right|^{2} d x-t^{q} \frac{c_{1}}{q} \varepsilon^{-2 \alpha} \int_{\mathbb{R}^{N}}\left|\psi_{\varepsilon, \delta}(x)\right|^{q} d x \\
= & \varepsilon^{N-2 \alpha}\left\{\frac{t^{2}}{2} \int_{\mathbb{R}^{2 N}} \frac{\left|\psi_{\delta}(x)-e^{\mathrm{i}(x-y) \cdot A\left(\frac{\varepsilon x+\varepsilon y}{2}\right)} \psi_{\delta}(y)\right|^{2}}{|x-y|^{N+2 \alpha}} d x d y+\frac{t^{2}}{2} \int_{\mathbb{R}^{N}} V(\varepsilon x)\left|\psi_{\delta}(x)\right|^{2} d x\right. \\
& \left.-t^{q} \frac{c_{1}}{q} \int_{\mathbb{R}^{N}}\left|\psi_{\delta}(x)\right|^{q} d x\right\}=: \varepsilon^{N-2 \alpha} J_{\varepsilon}\left(t \psi_{\delta}\right) .
\end{aligned}
$$

Now it is easy to see that assumption $q>2$ implies there exists $t_{0}>0$ such that

$$
I_{\varepsilon}\left(t_{0} \psi_{\varepsilon, \delta}\right) \leq \varepsilon^{N-2 \alpha} J_{\varepsilon}\left(t_{0} \psi_{\delta}\right)<0 .
$$

This finishes the proof.

Let $\psi_{\delta}(x)=e^{\mathrm{i} A(0) \cdot x} \phi_{\delta}(x)$, where $\phi_{\delta}$ is as in the proof of Lemma 3.5. Then, we have the following

Lemma 3.6 (Norm estimate). For any $\delta>0$ there exists $\varepsilon_{0}=\varepsilon_{0}(\delta)>0$ such that

$$
\int_{\mathbb{R}^{2 N}} \frac{\left|\psi_{\delta}(x)-e^{\mathrm{i}(x-y) \cdot A\left(\frac{\varepsilon x+\varepsilon y}{2}\right)} \psi_{\delta}(y)\right|^{2}}{|x-y|^{N+2 \alpha}} d x d y \leq C \delta^{\frac{2 N-(N-2 \alpha) q}{q}}+\frac{1}{1-\alpha} \delta^{2 \alpha}+\frac{4}{\alpha} \delta^{2 \alpha},
$$

for all $0<\varepsilon<\varepsilon_{0}$, for come constant $C>0$ depending only on $[\phi]_{\alpha, 0}$. 
Proof. For any $\delta>0$, we have

$$
\begin{aligned}
& \int_{\mathbb{R}^{2 N}} \frac{\left|\psi_{\delta}(x)-e^{\mathrm{i}(x-y) \cdot A\left(\frac{\varepsilon x+\varepsilon y}{2}\right)} \psi_{\delta}(y)\right|^{2}}{|x-y|^{N+2 \alpha}} d x d y \\
= & \int_{\mathbb{R}^{2 N}} \frac{\left|e^{\mathrm{i} A(0) \cdot x} \phi_{\delta}(x)-e^{\mathrm{i}(x-y) \cdot A\left(\frac{\varepsilon x+\varepsilon y}{2}\right)} e^{\mathrm{i} A(0) \cdot y} \phi_{\delta}(y)\right|^{2}}{|x-y|^{N+2 \alpha}} d x d y \\
\leq & 2 \int_{\mathbb{R}^{2 N}} \frac{\left|\phi_{\delta}(x)-\phi_{\delta}(y)\right|^{2}}{|x-y|^{N+2 \alpha}} d x d y+2 \int_{\mathbb{R}^{2 N}} \frac{\left|\phi_{\delta}(y)\right|^{2}\left|e^{\mathrm{i}(x-y) \cdot\left(A(0)-A\left(\frac{\varepsilon x+\varepsilon y}{2}\right)\right)}-1\right|^{2}}{|x-y|^{N+2 \alpha}} d x d y .
\end{aligned}
$$

Next we will estimate the second term in the above inequality. Notice that

$$
\left|e^{\mathrm{i}(x-y) \cdot\left(A(0)-A\left(\frac{\varepsilon x+\varepsilon y}{2}\right)\right)}-1\right|^{2}=4 \sin ^{2}\left[\frac{(x-y) \cdot\left(A(0)-A\left(\frac{\varepsilon x+\varepsilon y}{2}\right)\right)}{2}\right] .
$$

For any $y \in B_{r_{\delta}}$, if $|x-y| \leq \frac{1}{\delta}\left\|\phi_{\delta}\right\|_{L^{2}}^{\frac{1}{\alpha}}$, then $|x| \leq r_{\delta}+\frac{1}{\delta}\left\|\phi_{\delta}\right\|_{L^{2}}^{\frac{1}{\alpha}}$. Hence, we have

$$
\left|\frac{\varepsilon x+\varepsilon y}{2}\right| \leq \frac{\varepsilon}{2}\left(2 r_{\delta}+\frac{1}{\delta}\left\|\phi_{\delta}\right\|_{L^{2}}^{\frac{1}{\alpha}}\right) \text {. }
$$

Since $A: \mathbb{R}^{N} \rightarrow \mathbb{R}^{N}$ is continuous, there exists $\varepsilon_{0}>0$ such that for any $0<\varepsilon<\varepsilon_{0}$,

$$
\left|A(0)-A\left(\frac{\varepsilon x+\varepsilon y}{2}\right)\right| \leq \delta\left\|\phi_{\delta}\right\|_{L^{2}}^{-\frac{1}{\alpha}}, \quad \text { for }|y| \leq r_{\delta} \text { and }|x| \leq r_{\delta}+\frac{1}{\delta}\left\|\phi_{\delta}\right\|_{L^{2}}^{\frac{1}{\alpha}} .
$$

which implies

$$
\left|e^{\mathrm{i}(x-y) \cdot\left(A(0)-A\left(\frac{\varepsilon x+\varepsilon y}{2}\right)\right)}-1\right|^{2} \leq|x-y|^{2} \delta^{2}\left\|\phi_{\delta}\right\|_{L^{2}}^{-\frac{2}{\alpha}} .
$$

For all $\delta>0$ and $y \in B_{r_{\delta}}$, let us define

$$
M_{\delta, y}:=\left\{x \in \mathbb{R}^{N}:|x-y| \leq \frac{1}{\delta}\left\|\phi_{\delta}\right\|_{L^{2}}^{\frac{1}{\alpha}}\right\} .
$$

Then gathering the above facts, for all $0<\varepsilon<\varepsilon_{0}$, we have

$$
\begin{aligned}
& \int_{\mathbb{R}^{2 N}} \frac{\left|\phi_{\delta}(y)\right|^{2}\left|e^{\mathrm{i}(x-y) \cdot\left(A(0)-A\left(\frac{\varepsilon x+\varepsilon y}{2}\right)\right)}-1\right|^{2}}{|x-y|^{N+2 \alpha}} d x d y \\
= & \left(\int_{B_{r_{\delta}}}\left|\phi_{\delta}(y)\right|^{2} d y \int_{M_{\delta, y}}+\int_{B_{r_{\delta}}}\left|\phi_{\delta}(y)\right|^{2} d y \int_{\mathbb{R}^{N} \backslash M_{\delta, y}}\right) \frac{\left|e^{\mathrm{i}(x-y)\left(A(0)-A\left(\frac{\varepsilon x+\varepsilon y}{2}\right)\right)}-1\right|^{2}}{|x-y|^{N+2 \alpha}} d x \\
\leq & \int_{B_{r_{\delta}}}\left|\phi_{\delta}(y)\right|^{2} d y \int_{M_{\delta, y}} \frac{|x-y|^{2}}{|x-y|^{N+2 \alpha}} \delta^{2}\left\|\phi_{\delta}\right\|_{L^{2}}^{-\frac{2}{\alpha}} d x+\int_{B_{r_{\delta}}}\left|\phi_{\delta}(y)\right|^{2} d y \int_{\mathbb{R}^{N} \backslash M_{\delta, y}} \frac{4}{|x-y|^{N+2 \alpha}} d x \\
\leq & \frac{1}{2-2 \alpha} \delta^{2 \alpha}+\frac{4}{2 \alpha} \delta^{2 \alpha} .
\end{aligned}
$$

Combining the previous inequalities concludes the proof.

Let $t_{0}=t_{0}(\varepsilon, \delta)>0$ and $\psi_{\varepsilon, \delta}$ of Lemma 3.5. Then, we have the following

Lemma 3.7 (Mountain Pass geometry II). For any $\varepsilon>0$ and $\delta>0$, there exist

$$
d_{\varepsilon, \delta}>0 \quad \text { and } \quad 0<\rho_{\varepsilon, \delta}<\left\|t_{0} \psi_{\varepsilon, \delta}\right\|_{\varepsilon},
$$

with $I_{\varepsilon}(u) \geq d_{\varepsilon, \delta}$ for $u \in X_{\varepsilon}$ with $\|u\|_{\varepsilon}=\rho_{\varepsilon, \delta}$ and $I_{\varepsilon}(u)>0$ for any $u \in X_{\varepsilon} \backslash\{0\}$ with $\|u\|_{\varepsilon}<\rho_{\varepsilon, \delta}$. 
Proof. By $\left(f_{1}\right)$ and $\left(f_{2}\right)$, for any $\tau>0$, there exists $C(\tau)>0$ such that

$$
|F(x, t)| \leq \tau t^{2}+C(\tau)|t|^{2_{\alpha}^{*}} .
$$

For any $u \in X_{\varepsilon}$, from Proposition 2.2, we derive

$$
\begin{aligned}
I_{\varepsilon}(u) & \geq \frac{1}{2}\|u\|_{\varepsilon}^{2}-\tau \varepsilon^{-2 \alpha}\|u\|_{L^{2}}^{2}-C(\tau) \varepsilon^{-2 \alpha}\|u\|_{L^{2 *}}^{2_{\alpha}^{*}}-\varepsilon^{-2 \alpha} \frac{K_{1}}{2_{\alpha}^{*}}\|u\|_{L^{2}}^{2_{\alpha}^{*}} \\
& \geq \frac{1}{2}\|u\|_{\varepsilon}^{2}-\tau \varepsilon^{-2 \alpha} c^{2}(\varepsilon)\|u\|_{\varepsilon}^{2}-C(\varepsilon)\|u\|_{\varepsilon}^{2_{\alpha}^{*}},
\end{aligned}
$$

where $c(\varepsilon)>0$ is the embedding constant of $\left(X_{\varepsilon},\|\cdot\|_{\varepsilon}\right) \hookrightarrow L^{2}\left(\mathbb{R}^{N}, \mathbb{C}\right)$. Letting $\tau<\frac{\varepsilon^{2 \alpha}}{4 c^{2}(\varepsilon)}$, we get

$$
I_{\varepsilon}(u) \geq \frac{1}{4}\|u\|_{\varepsilon}^{2}-C(\varepsilon)\|u\|_{\varepsilon}^{2^{*}} .
$$

Then, there exist $d_{\varepsilon, \delta}>0$ and $0<\rho_{\varepsilon, \delta}<\left\|t_{0} \psi_{\varepsilon, \delta}\right\|_{\varepsilon}$ such that $I_{\varepsilon}(u) \geq d_{\varepsilon, \delta}$ for $u \in X_{\varepsilon}$ with $\|u\|_{\varepsilon}=\rho_{\varepsilon, \delta}$ and $I_{\varepsilon}(u)>0$ for any $u \in X_{\varepsilon} \backslash\{0\}$ with $\|u\|_{\varepsilon}<\rho_{\varepsilon, \delta}$.

Proposition 3.1 (Sobolev constant bounds). There exists $\mathcal{S}_{\alpha}, \mathcal{S}^{\alpha}>0$ independent of $\varepsilon$ with

$$
\mathcal{S}_{\alpha} \leq S_{\alpha}^{\varepsilon} \leq \mathcal{S}^{\alpha}, \quad \text { for every } \varepsilon>0 .
$$

In particular, with reference to Lemma 3.4, the Palais-Smale for $I_{\varepsilon}$ holds for

$$
-\infty<c<C_{0} \varepsilon^{N-2 \alpha}, \quad C_{0}:=\left(\frac{\mathcal{S}_{\alpha}}{c_{2}+K_{1}}\right)^{\frac{2_{\alpha}^{*}}{2 *-2}} \frac{\left(2_{\alpha}^{*}-\mu\right) K_{0}}{\mu 2_{\alpha}^{*}} .
$$

Proof. By virtue of the pointwise diamagnetic inequality [13, Remark 3.2]

$$
|| u(x)|-| u(y)|| \leq\left|u(x)-e^{\mathrm{i}(x-y) \cdot A_{\varepsilon}\left(\frac{x+y}{2}\right)} u(y)\right|, \quad \text { for a.e. } x, y \in \mathbb{R}^{N} \text { and all } \varepsilon>0,
$$

we have

$$
S_{\alpha}^{\varepsilon}=\inf _{u \in D_{A_{\varepsilon}}^{\alpha}\left(\mathbb{R}^{N}\right) \backslash\{0\}} \frac{[u]_{\alpha, A_{\varepsilon}}^{2}}{\|u\|_{L^{2 *}}^{2}} \geq \inf _{D_{A_{\varepsilon}}^{\alpha}\left(\mathbb{R}^{N}\right) \backslash\{0\}} \frac{\left(\int_{\mathbb{R}^{2 N}} \frac{\|u(x)|-| u(y)\|^{2}}{|x-y|^{N+2 \alpha}} d x d y\right)^{1 / 2}}{\|u\|_{L^{2}}^{2}} \geq \mathcal{S}_{\alpha},
$$

where $\mathcal{S}_{\alpha}>0$ is the Sobolev constant for the embedding $D^{\alpha}\left(\mathbb{R}^{N}\right) \hookrightarrow L^{2_{\alpha}^{*}}\left(\mathbb{R}^{N}\right)$. Concerning the opposite inequality, fix $\varphi \in C_{c}^{\infty}\left(\mathbb{R}^{N}\right) \backslash\{0\}$ with $\|\varphi\|_{L^{2_{\alpha}^{*}}}=1$ and use the function

$$
x \mapsto \varphi\left(\frac{x}{\varepsilon}\right) e^{\mathrm{i} A(0) \cdot \frac{x}{\varepsilon}}
$$

in the definition of $S_{\alpha}^{\varepsilon}$. We have

$$
S_{\alpha}^{\varepsilon} \leq \int_{\mathbb{R}^{2 N}} \frac{\left|\varphi(x)-e^{\mathrm{i}(x-y) \cdot\left(A\left(\frac{\varepsilon x+\varepsilon y}{2}\right)-A(0)\right)} \varphi(y)\right|^{2}}{|x-y|^{N+2 \alpha}} d x d y \leq \mathbb{I}_{1}+\mathbb{I}_{2},
$$

where

$$
\mathbb{I}_{1}=2 \int_{\mathbb{R}^{2 N}} \frac{|\varphi(x)-\varphi(y)|^{2}}{|x-y|^{N+2 \alpha}} d x d y, \quad \mathbb{I}_{2}=2 \int_{\mathbb{R}^{2 N}} \frac{|\varphi(y)|^{2}\left|e^{\mathrm{i}(x-y) \cdot\left(A(0)-A\left(\frac{\varepsilon x+\varepsilon y}{2}\right)\right)}-1\right|^{2}}{|x-y|^{N+2 \alpha}} d x d y .
$$

It is sufficient to estimate $\mathbb{I}_{2}$ from above independently of $\varepsilon>0$. If $K$ is the support of $\varphi$, let

$$
M_{y}:=\left\{x \in \mathbb{R}^{N}:|x-y| \leq 1\right\}, \quad y \in K,
$$


Taking into account (3.13), for some $C>0$ independent of $\varepsilon$, we have

$$
\begin{aligned}
\mathbb{I}_{2} & =\left(\int_{K}|\varphi(y)|^{2} d y \int_{M_{y}}+\int_{K}|\varphi(y)|^{2} d y \int_{\mathbb{R}^{N} \backslash M_{y}}\right) \frac{\left|e^{\mathrm{i}(x-y)\left(A(0)-A\left(\frac{\varepsilon x+\varepsilon y}{2}\right)\right)}-1\right|^{2}}{|x-y|^{N+2 \alpha}} d x \\
& \leq C \int_{K}|\varphi(y)|^{2} d y \int_{M_{y}} \frac{|x-y|^{2}}{|x-y|^{N+2 \alpha}} d x+C \int_{K}|\varphi(y)|^{2} d y \int_{\mathbb{R}^{N} \backslash M_{y}} \frac{1}{|x-y|^{N+2 \alpha}} d x=: \mathcal{S}^{\alpha}>0,
\end{aligned}
$$

concluding the proof.

\section{Proof of Theorem 1.1 CONCluded}

We shall prove that there exists $\varepsilon_{0}>0$ such that for any $\varepsilon \in\left(0, \varepsilon_{0}\right)$, problem (1.1) admits a solution $u_{\varepsilon} \in X_{\varepsilon}$ close to the trivial one in $X_{\varepsilon}$ for the norm $\|\cdot\|_{X_{\varepsilon}}$. For any $t>0$, from Lemma 3.6 we have

$$
\begin{aligned}
I_{\varepsilon}\left(t \psi_{\varepsilon, \delta}\right) & \leq c_{1}^{-\frac{2}{q-2}} \frac{q-2}{2 q} \varepsilon^{N-2 \alpha}\left(\int_{\mathbb{R}^{2 N}} \frac{\left|\psi_{\delta}(x)-e^{\mathrm{i}(x-y) \cdot A\left(\frac{\varepsilon x+\varepsilon y}{2}\right)} \psi_{\delta}(y)\right|^{2}}{|x-y|^{N+2 \alpha}} d x d y+\int_{\mathbb{R}^{N}} V(\varepsilon x)\left|\psi_{\delta}\right|^{2} d x\right)^{\frac{q}{q-2}} \\
& \leq c_{1}^{-\frac{2}{q-2}} \frac{q-2}{2 q} \varepsilon^{N-2 \alpha}\left(C \delta^{\frac{2 N-(N-2 \alpha) q}{q}}+\frac{1}{1-\alpha} \delta^{2 \alpha}+\frac{4}{\alpha} \delta^{2 \alpha}+\int_{\mathbb{R}^{N}} V(\varepsilon x)\left|\psi_{\delta}\right|^{2} d x\right)^{\frac{q}{q-2}} .
\end{aligned}
$$

Choose now $\delta>0$, depending only upon $N, \alpha, f, A, K$, such that

$$
c_{1}^{-\frac{2}{q-2}} \frac{q-2}{2 q}\left(C \delta^{\frac{2 N-(N-2 \alpha) q}{q}}+\frac{1}{1-\alpha} \delta^{2 \alpha}+\frac{4}{\alpha} \delta^{2 \alpha}+\delta\right)^{\frac{q}{q-2}}<C_{0},
$$

where $C_{0}$ is defined in (3.14). Since $V(x) \rightarrow 0$ as $|x| \rightarrow 0$, there is $x_{0, \delta}>0$ with

$$
|V(x)|<\frac{\delta}{\left\|\psi_{\delta}\right\|_{L^{2}}^{2}}, \quad \text { for all }|x|<x_{0, \delta} .
$$

We take $\varepsilon_{1}=\min \left\{\varepsilon_{0}, \frac{x_{0, \delta}}{r_{\delta}}\right\}$. Then, for any $\varepsilon<\varepsilon_{1}$, we have

$$
\int_{B_{r_{\delta}}} V(\varepsilon x)\left|\psi_{\delta}(x)\right|^{2} d x<\delta
$$

From the above estimate, we obtain

$$
\max _{t \geq 0} I_{\varepsilon}\left(t \psi_{\varepsilon, \delta}\right)<C_{0} \varepsilon^{N-2 \alpha}
$$

Denote, for every $\varepsilon>0$,

$$
c_{\varepsilon}:=\inf _{\gamma \in \Gamma} \max _{t \in[0,1]} I_{\varepsilon}(\gamma(t)), \quad \Gamma_{\varepsilon}:=\left\{\gamma \in C\left([0,1], X_{\varepsilon}\right): \gamma(0)=0, \gamma(1)=t_{0} \psi_{\varepsilon, \delta}\right\} .
$$

Then, we have

$$
\inf _{\|u\|_{\varepsilon}=\rho_{\varepsilon, \delta}} I_{\varepsilon}(u)>I_{\varepsilon}(0)>I_{\varepsilon}\left(t_{0} \psi_{\varepsilon, \delta}\right)
$$

and, by using the curve $\gamma(t)(x):=t t_{0} \psi_{\varepsilon, \delta}(x)$ of $\Gamma_{\varepsilon}$, we get

$$
0<d_{\varepsilon, \delta} \leq c_{\varepsilon} \leq \max _{t \in[0,1]} I_{\varepsilon}\left(t t_{0} \psi_{\varepsilon, \delta}\right) \leq \max _{t \geq 0} I_{\varepsilon}\left(t \psi_{\varepsilon, \delta}\right)<C_{0} \varepsilon^{N-2 \alpha} .
$$

By the Mountain Pass Theorem, there exists a sequence $\left\{u_{n}\right\}_{n \in \mathbb{N}} \subset X_{\varepsilon}$ such that

$$
I_{\varepsilon}\left(u_{n}\right) \rightarrow c_{\varepsilon} \text { and } I_{\varepsilon}^{\prime}\left(u_{n}\right) \rightarrow 0 \text { in } X_{\varepsilon}^{*} \text {, as } n \rightarrow \infty .
$$


By Proposition 3.1, there is a subsequence $\left\{u_{n_{j}}\right\}_{j \in \mathbb{N}}$ such that $u_{n_{j}} \rightarrow u_{\varepsilon}$ in $X_{\varepsilon}$. Thus $I_{\varepsilon}\left(u_{\varepsilon}\right)=c_{\varepsilon}$ and $I_{\varepsilon}^{\prime}\left(u_{\varepsilon}\right)=0$, namely $u_{\varepsilon}$ is a nontrivial weak solution of (1.1). Besides, from (4.1) we get

$$
\begin{aligned}
C_{0} \varepsilon^{N-2 \alpha}>c_{\varepsilon} & =I_{\varepsilon}\left(u_{\varepsilon}\right)-\frac{1}{\mu}\left\langle I_{\varepsilon}^{\prime}\left(u_{\varepsilon}\right), u_{\varepsilon}\right\rangle \\
& \geq\left(\frac{1}{2}-\frac{1}{\mu}\right)\left[u_{\varepsilon}\right]_{\alpha, A_{\varepsilon}}^{2}+\left(\frac{1}{2}-\frac{1}{\mu}\right) \varepsilon^{-2 \alpha} \int_{\mathbb{R}^{N}} V(x)\left|u_{\varepsilon}\right|^{2} d x,
\end{aligned}
$$

which implies that

$$
\left[u_{\varepsilon}\right]_{\alpha, A_{\varepsilon}}^{2}<\frac{2 C_{0} \mu}{\mu-2} \varepsilon^{N-2 \alpha}, \quad \int_{\mathbb{R}^{N}} V(x)\left|u_{\varepsilon}\right|^{2} d x<\frac{2 C_{0} \mu}{\mu-2} \varepsilon^{N} .
$$

Then $u_{\varepsilon} \rightarrow 0$ in $X_{\varepsilon}$ for the norm $\|\cdot\|_{X_{\varepsilon}}$, as $\varepsilon \rightarrow 0$.

\section{Some Results Without Magnetic FIELD}

In this Section, we consider the existence of solutions for (1.1) without magnetic field, i.e. $A \equiv 0$. We first establish the existence of $m$ pairs of solutions of via the Ljusternik-Schnirelmann theory of critical points. Let $\Sigma\left(X_{\varepsilon}\right)$ be the family of sets $F \subseteq \Sigma\left(X_{\varepsilon}\right) \backslash\{0\}$ such that $F$ is closed in $X_{\varepsilon}$ and symmetric with respect to 0, i.e. $x \in F$ implies $-x \in F$. For $F \in \Sigma\left(X_{\varepsilon}\right)$, we define the genus of $F$ to be $k$, denoted by $\operatorname{gen}(F)=k$, if there is a continuous and odd map $\psi: F \rightarrow \mathbb{R}^{k} \backslash\{0\}$ and $k$ is the smallest integer with this property. The definition of genus here, which was by Coffman [6], is equivalent with the Krasnoselski original genus. Denote by $\Gamma_{*}$ the set of all odd homeomorphisms $g \in C\left(X_{\varepsilon}, X_{\varepsilon}\right)$ such that $g(0)=0$ and $g\left(B_{1}\right) \subseteq\left\{u \in X_{\varepsilon}: I_{\varepsilon}(u) \geq 0\right\}$. We denote by $\Gamma_{m}$ the set of all compact subsets $F$ of $X_{\varepsilon}$ which are symmetric with respect to the origin and satisfies gen $\left(F \cap g\left(\partial B_{1}\right)\right) \geq m$ for any $g \in \Gamma_{*}$. We refer to [7] for more details.

Theorem 5.1. Assume that hypotheses $\left(V_{1}\right)-\left(V_{2}\right),\left(f_{1}\right)-\left(f_{4}\right)$ and $(K)$ are fulfilled. If the subcritical nonlinearity $f(x, t)$ is odd in $t$, for any $m \in \mathbb{N}$ there exist $\varepsilon_{m}>0$ such that for any $\varepsilon \in\left(0, \varepsilon_{m}\right)$, problem (1.1) has at least $m$ pairs of nontrivial weak solutions in $X_{\varepsilon}$.

Proof. As in Lemma 3.5, for any $m \in \mathbb{N}$, we can take $\phi_{\delta}^{j} \in C_{0}^{\infty}\left(\mathbb{R}^{N}\right)$ such that, for any $j=$ $1 \ldots, m$,

$$
\operatorname{supp} \phi_{\delta}^{j} \subset B_{r_{m, \delta}}\left(x_{j, \delta}\right), \quad\left\|\phi_{\delta}^{j}\right\|_{L^{q}}=1, \quad\left[\phi_{\delta}^{j}\right]_{\alpha, 0}<C \delta^{\frac{2 N-(N-2 \alpha) q}{q}},
$$

with $B_{r_{m, \delta}}\left(x_{i, \delta}\right) \cap B_{r_{m, \delta}}\left(x_{j, \delta}\right)=\emptyset$, for any $i \neq j$. Set $e_{\varepsilon, \delta}^{j}(x)=\phi_{\delta}^{j}\left(\varepsilon^{-1} x\right)$. Thus

$$
\int_{\mathbb{R}^{2 N}} \frac{\left|e_{\varepsilon, \delta}^{j}(x)-e_{\varepsilon, \delta}^{j}(y)\right|^{2}}{|x-y|^{N+2 \alpha}} d x d y<C \delta^{\frac{2 N-(N-2 \alpha) q}{q}} \varepsilon^{N-2 \alpha}, \quad \int_{\mathbb{R}^{N}}\left|e_{\varepsilon, \delta}^{j}\right|^{q} d x=\varepsilon^{N} .
$$

Define $m$-dimensional subspace $F_{m}^{\varepsilon, \delta}:=\operatorname{span}\left\{e_{\varepsilon, \delta}^{j}\right\}_{j=1, \ldots, m}$. For any $\delta>0$ with

$$
m^{\frac{3 q-2}{q-2}} c_{1}^{-\frac{2}{q-2}} \frac{q-2}{2 q}\left(C \delta^{\frac{2 N-(N-2 \alpha) q}{q}}+\delta\right)^{\frac{q}{q-2}}<C_{0} .
$$

Let now for any $j=1, \ldots, m$ radii $R_{j, \delta}>0$ with $B_{r_{m, \delta}}\left(x_{j, \delta}\right) \subset B_{R_{j, \delta}}(0)$. Therefore, since $V(x) \rightarrow 0$ as $|x| \rightarrow 0$, there is $x_{j, \delta}>0$ with

$$
|V(x)|<\frac{\delta}{\left\|\phi_{\delta}^{j}\right\|_{L^{2}}^{2}}, \quad \text { for all }|x|<x_{j, \delta} .
$$

Then, for any $\varepsilon<\frac{x_{j, \delta}}{R_{j, \delta}}$, we have

$$
\int_{B_{r_{m, \delta}\left(x_{j}\right)}} V(\varepsilon x)\left|\phi_{\delta}^{j}(x)\right|^{2} d x \leq \int_{B_{R_{j, \delta}(0)}} V(\varepsilon x)\left|\phi_{\delta}^{j}(x)\right|^{2} d x<\delta .
$$


Then, for any $\varepsilon<\min _{j=1, \ldots, m}\left\{\frac{x_{j, \delta}}{R_{j, \delta}}\right\}$ and $u \in F_{m}^{\varepsilon, \delta}$ with $u=\sum_{j=1}^{m} t_{j} e_{\varepsilon, \delta}^{j}$, by $\left(f_{4}\right)$ we get

$$
\begin{aligned}
& I_{\varepsilon}(u) \leq \frac{1}{2} \int_{\mathbb{R}^{2 N}} \frac{|u(x)-u(y)|^{2}}{|x-y|^{N+2 \alpha}} d x d y+\frac{\varepsilon^{-2 \alpha}}{2} \int_{\mathbb{R}^{N}} V(x) u^{2} d x-\frac{c_{1} \varepsilon^{-2 \alpha}}{q} \int_{\mathbb{R}^{N}}|u|^{q} d x \\
\leq & \sum_{j=1}^{m}\left(m^{2} \frac{t_{j}^{2}}{2} \int_{\mathbb{R}^{2 N}} \frac{\left|e_{\varepsilon, \delta}^{j}(x)-e_{\varepsilon, \delta}^{j}(y)\right|^{2}}{|x-y|^{N+2 \alpha}} d x d y+\varepsilon^{-2 \alpha} m^{2} \frac{t_{j}^{2}}{2} \int_{\mathbb{R}^{N}} V(x)\left|e_{\varepsilon, \delta}^{j}\right|^{2} d x-\varepsilon^{-2 \alpha} \frac{c_{1}}{q} t_{j}^{q} \int_{\mathbb{R}^{N}}\left|e_{\varepsilon, \delta}^{j}\right|^{q} d x\right) \\
\leq & \varepsilon^{N-2 \alpha} \sum_{j=1}^{m}\left(\left[m^{2} \int_{\mathbb{R}^{2 N}} \frac{\left|\phi_{\delta}^{j}(x)-\phi_{\delta}^{j}(y)\right|^{2}}{|x-y|^{N+2 \alpha}} d x d y+m^{2} \int_{\mathbb{R}^{N}} V(\varepsilon x)\left|\phi_{\delta}^{j}\right|^{2} d x\right] \frac{t_{j}^{2}}{2}-c_{1} \frac{t_{j}^{q}}{q}\right) \\
\leq & m^{\frac{3 q-2}{q-2}} c_{1}^{-\frac{2}{q-2}} \frac{q-2}{2 q}\left(C \delta^{\frac{2 N-(N-2 \alpha) q}{q}}+\delta\right)^{\frac{q}{q-2}} \varepsilon^{N-2 \alpha}<C_{0} \varepsilon^{N-2 \alpha} .
\end{aligned}
$$

Since $\operatorname{dim}\left(F_{m}^{\varepsilon, \delta}\right)<\infty,\|\cdot\|_{L^{q}\left(\mathbb{R}^{N}\right)}$ and $\|\cdot\|_{\varepsilon}$ are equivalent. Then $I_{\varepsilon}(u) \rightarrow-\infty$, as $u \in F_{m}^{\varepsilon, \delta}$ with $\|u\|_{\varepsilon} \rightarrow \infty$. For any $1 \leq j \leq m$, let

$$
c_{\varepsilon}^{j}=\inf _{F \in \Gamma_{m}} \max _{u \in F} I_{\varepsilon}(u)
$$

we have

$$
d_{\varepsilon} \leq c_{\varepsilon}^{1} \leq c_{\varepsilon}^{2} \leq \cdots \leq c_{\varepsilon}^{m} \leq \sup _{u \in F_{m}^{\varepsilon, \delta}} I_{\varepsilon}(u) \leq C_{0} \varepsilon^{N-2 \alpha} .
$$

From Proposition 3.1, $I_{\varepsilon}$ satisfies (PS) $)_{c_{\varepsilon}^{j}}$ condition. Thus, $c_{\varepsilon}^{j}$ is a critical value of $I_{\varepsilon}$ and $u_{\varepsilon, j}$ is a critical point of $I_{\varepsilon}$ with $I_{\varepsilon}\left(u_{\varepsilon, j}\right)=c_{\varepsilon}^{j}$. As $f(x, t)$ is odd in $t$, we derive that $-u_{\varepsilon, j}$ is also a critical point of $I_{\varepsilon}$. Then $I_{\varepsilon}$ has at least $m$ pairs of nontrivial solutions.

Finally, we verify that problem (1.1) has one pair of sign-changing solutions. Let $g: \mathbb{R}^{N} \rightarrow \mathbb{R}^{N}$ be an orthogonal involution. Then the action of $g$ on $X$ is defined by

$$
g u(x)=-u(g x), \quad \text { for any } u \in X_{\varepsilon} .
$$

If $V(g x)=V(x), h(g x)=h(x)$ and $f(g x, t)=f(x, t)$, it is easy to verify that $I_{\varepsilon}$ is $g$-invariant, i.e. $I_{\varepsilon}(g u)=I_{\varepsilon}(u)$ and $I_{\varepsilon}^{\prime}(g u)=g I_{\varepsilon}^{\prime}(u)$. The subspace of $g$-invariant functions is defined by

$$
X_{g}=\left\{u \in X_{\varepsilon}: g u=u\right\}
$$

Then the critical points of $\widetilde{I}_{\varepsilon}=\left.I_{\varepsilon}\right|_{X_{g}}$ are critical points of $I_{\varepsilon}$. Therefore, it suffices to prove the existence of critical points for $\widetilde{I}_{\varepsilon}$ on $X_{g}$. As a consequence, we obtain the following result:

Theorem 5.2. Assume that $\left(V_{1}\right)-\left(V_{2}\right),\left(f_{1}\right)-\left(f_{4}\right)$ and $(K)$ are satisfied. If the nonlinearity $f(x, t)$ is odd in $t$ and there is an orthogonal involution $g$ such that $V(g x)=V(x), h(g x)=h(x)$ and $f(g x, t)=f(x, t)$, then there exist $\varepsilon^{*}>0$ such that for any $\varepsilon \in\left(0, \varepsilon^{*}\right)$, problem (1.1) has at least one pair of sign-changing weak solutions in $X$.

Proof. Note that for any $\phi \in C_{0}^{\infty}\left(\mathbb{R}^{N}\right), \widetilde{\phi}=\frac{\phi+g \phi}{2} \in C_{0}^{\infty}\left(\mathbb{R}^{N}\right) \cap X_{g}$. One could verify that

$$
\inf \left\{\int_{\mathbb{R}^{2 N}} \frac{|\phi(x)-\phi(y)|^{2}}{|x-y|^{N+2 \alpha}} d x d y: \phi \in C_{0}^{\infty}\left(\mathbb{R}^{N}\right) \cap X_{g} \text { with }\|\phi\|_{L^{q}\left(\mathbb{R}^{N}\right)}=1\right\}=0 .
$$

Then, it is readily seen that $\widetilde{I}_{\varepsilon}$ has a Mountain Pass geometry: for any $\varepsilon>0$ and $\delta>0$ :

(1) there exists $\widetilde{t}_{0}>0$ and $\widetilde{e}_{\varepsilon, \delta} \in X_{g}$ such that $\widetilde{I}_{\varepsilon}\left(\widetilde{t}_{0} \widetilde{e}_{\varepsilon, \delta}\right)<0$. 
(2) there exists $\widetilde{d}_{\varepsilon}>0$ and $0<\widetilde{\rho}_{\varepsilon}<\left\|t_{0} \widetilde{e}_{\varepsilon, \delta}\right\|_{\varepsilon}$ such that $\widetilde{I}_{\varepsilon}(u) \geq \widetilde{d}_{\varepsilon}$ for any $u \in X_{g}$ with $\|u\|_{\varepsilon}=\widetilde{\rho}_{\varepsilon}$ and $\widetilde{I}_{\varepsilon}(u)>0$ for any $u \in X_{g}$ with $\|u\|_{\varepsilon}<\widetilde{\rho}_{\varepsilon}$. Denote

$$
\widetilde{c}_{\varepsilon}=\inf _{\gamma \in \Gamma} \max _{t \in[0,1]} \widetilde{I}_{\varepsilon}(\gamma(t)),
$$

where $\Gamma=\left\{\gamma \in C\left([0,1], X_{g}\right): \gamma(0)=0, \gamma(1)=\widetilde{t}_{0} \widetilde{e}_{\varepsilon, \delta}\right\}$. Then, there is $\varepsilon^{*}>0$ with, for $0<\varepsilon<\varepsilon^{*}$,

$$
\begin{gathered}
\inf _{\|u\|_{\varepsilon}=\widetilde{\rho}_{\varepsilon}} \widetilde{I}_{\varepsilon}(u)>\widetilde{I}_{\varepsilon}(0)>\widetilde{I}_{\varepsilon}\left(\widetilde{t}_{0} \widetilde{e}_{\varepsilon, \delta}\right), \\
0<\widetilde{d}_{\varepsilon} \leq \widetilde{c}_{\varepsilon} \leq \widetilde{I}_{\varepsilon}\left(t \widetilde{t}_{0} \widetilde{e}_{\varepsilon, \delta}\right) \leq c_{1}^{-\frac{2}{q-2}} \frac{q-2}{2 q}\left(C \delta^{\frac{2 N-(N-2 \alpha) q}{q}}+\delta\right)^{\frac{q}{q-2}} \varepsilon^{N-2 \alpha}<C_{0} \varepsilon^{N-2 \alpha} .
\end{gathered}
$$

where $C_{0}$ is as in Proposition 3.1. Then there exists $\widetilde{u}_{\varepsilon} \in X_{g}$ such that $\widetilde{I}_{\varepsilon}^{\prime}\left(\widetilde{u}_{\varepsilon}\right)=0$. Then, $\widetilde{u}_{\varepsilon}$ is a critical point of $I_{\varepsilon}$ and $\widetilde{u}_{\varepsilon}(x)=g \widetilde{u}_{\varepsilon}(x)=-\widetilde{u}_{\varepsilon}(g x)$. It is easy to show that $\widetilde{u}_{\varepsilon}(g x)$ is also a critical point of $I_{\varepsilon}$ and $\widetilde{u}_{\varepsilon}(x), \widetilde{u}_{\varepsilon}(g x)$ change sign.

\section{REFERENCES}

[1] D. Applebaum, Lévy processes-from probalility to finance and quantum groups. Notices Amer. Math. Soc. 51 (2004), 1336-1347. 1

[2] G. Autuori, P. Pucci, Elliptic problems involving the fractional Laplacian in $\mathbb{R}^{N}$, J. Differential Equations 255 (2013) 2340-2362. 1

[3] H. Brézis, L. Nirenberg, Positive solutions of nonlinear elliptic equations involving critical Sobolev exponents, Comm. Pure Appl. Math. 36 (1983), 437-477. 5

[4] J. Byeon, Z.-Q. Wang, Standing waves with a critical frequency for nonlinear Schrodinger equations, Arch. Ration. Mech. Anal. 165 (2002), 295-316. 3

[5] J. Byeon, Z.-Q. Wang, Standing waves with a critical frequency for nonlinear Schrödinger equations, II, Calc. Var. Partial Differential Equations 18 (2003), 207-219. 3

[6] C.V. Coffman, A minimum-maximum principle for a class of non-linear integral equations, J. Anal. Math. 22 (1969), 392-419. 17

[7] J. Chabrowski, Variational Methods for Potential Operator Equations, de Gruyter Studies in Mathematics 24, de Gruyter (1997). 17

[8] X.J. Chang, Z.-Q. Wang, Ground state of scalar field equations involving a fractional Laplacian with general nonlinearity, Nonlinearity 26 (2013), 479-494. 1

[9] G.Y. Chen, Y.Q. Zheng, Concentration phenomenon for fractional nonlinear Schrödinger equations, Comm. Pure Appl. Anal. 13 (2014), 2359-2376. 2

[10] M. Cheng, Bound state for the fractional Schrödinger equation with unbounded potential, J. Math. Phys. 53, 043507 (2012). 2

[11] J. Dávila, M. del Pino, J.C. Wei, Concentrating standing waves for the fractional nonlinear Schrödinger equation, J. Differential Equations 256 (2014), 858-892. 2

[12] E. Di Nezza, G. Palatucci, E. Valdinoci, Hitchhiker's guide to the fractional Sobolev spaces. Bull. Sci. Math. 136 (2012), 521-573.

[13] P. d'Avenia, M. Squassina, Ground states for fractional magnetic operators, http://arxiv.org/abs/1601.042301,4,15

[14] Y. Ding, Z.-Q. Wang, Bound states of nonlinear Schrödinger equations with magnetic fields, Ann. Mat. Pura Appl. 190 (2011), 427-451. 2, 3

[15] Y. Ding, F. Lin, Solutions of perturbed schrödinger equations with critical nonlinearity, Calc. Var. Partial Differential Equations 30 (2007), 231-249. 3

[16] M. Esteban, P.L. Lions, Stationary solutions of nonlinear schrödinger equations with an external magnetic field, Partial differential equations and the calculus of variations, Vol. I, 401-449, Progr. Nonlinear Differential Equations Appl. 1, Birkhäuser Boston, Boston, MA, 1989. 1

[17] P. Felmer, A. Quaas, J. G. Tan, Positive solutions of the nonlinear schrödinger equation with the fractional Laplacian. Proc. Royal Soc. Edinb. A 142 (2012), 1237-1262. 1, 2

[18] A. Fiscella, E. Valdinoci, A critical Kirchhoff type problem involving a nonlocal operator, Nonlinear Anal. 94 (2014), 156-170. 1 
[19] T. Ichinose, Magnetic relativistic Schrödinger operators and imaginary-time path integrals, Mathematical physics, spectral theory and stochastic analysis, 247-297, Oper. Theory Adv. Appl. 232, Birkhäuser/Springer Basel AG, Basel, 2013. 1

[20] T. Ichinose, Essential selfadjointness of the Weyl quantized relativistic Hamiltonian, Ann. Inst. H. Poincaré Phys. Théor. 51 (1989), 265-297. 1

[21] T. Ichinose, H. Tamura, Imaginary-time path integral for a relativistic spinless particle in an electromagnetic field, Comm. Math. Phys. 105 (1986), 239-257. 1

[22] N. Laskin, Fractional quantum mechanics and Lévy path integrals, Phys. Lett. A 268 (2000), 298-305. 1, 2

[23] N. Laskin, Fractional Schrödinger equation, Phys. Rev. E 66, 056108 (2002). 1, 2

[24] C. Ledesma, Existence and concentration of solutions for a nonlinear fractional Schrödinger equations with steep potential well, Commun. Pure Appl. Anal. 15 (2016), 535-547. 2

[25] P. Pucci, M.Q. Xiang, B.L. Zhang, Multiple solutions for nonhomogeneous Schrödinger-Kirchhoff type equations involving the fractional $p$-Laplacian in $\mathbb{R}^{N}$, Calc. Var. Partial Differential Equations 54 (2015), 27852806. 1

[26] M. Reed, B. Simon, Methods of Modern Mathematical Physics, IV Analysis of Operators, Academic Press, London, 1978. 2

[27] S. Secchi, Ground states solutions for nonlinear fractional Schrödinger equations in $\mathbb{R}^{n}$, J. Math. Phys. 54 (2013), 031501. 2

[28] Z. Shen, F. Gao, On the existence of solutions for the critical fractional Laplacian equation in $\mathbb{R}^{N}$, Abstract Appl. Anal. 2014(2014) 1-10. 2

[29] X. Shang, J. Zhang, Ground states for fractional Schrödinger equations with critical growth, Nonlinearity 27 (2014), 187-207. 2

[30] X. D. Shang, J. H. Zhang, Concentrating solutions of nonlinear fractional Schrödinger equation with potentials, J. Differential Equations 258 (2015), 1106-1128. 2

[31] M. Squassina, Soliton dynamics for the nonlinear Schrödinger equation with magnetic field, Manuscripta Math. 130 (2009), 461-494. 2

[32] M. Squassina, B. Volzone, Bourgain-Brezis-Mironescu formula for magnetic operators, Comptes Rendus Mathematique, doi: 10.1016/j.crma.2016.04.013. 1, 3

[33] J. L. Vázquez, Nonlinear diffusion with fractional Laplacian operators, Nonlinear Partial Differential Equations, Abel Symposia 7 (2012) 271-298. 1

[34] X. Zhang, B.L. Zhang, D. Repovš, Existence and symmetry of solutions for critical fractional Schrödinger equations with bounded potentials, Nonlinear Anal. 142 (2016), 48-68. 2

[35] X. Zhang, B.L. Zhang, M.Q. Xiang, Ground states for fractional Schrödinger equations involving a critical nonlinearity, Adv. Nonlinear Anal. doi: 10.1515/anona-2015-0133. 2

(Zhang Binlin) Department of Mathematics

HeilongJiang Institute of TEChNOLOGy

HARbin 150050, P.R. China

E-mail address: zhangbinlin2012@163.com

(Marco Squassina) Dipartimento di Matematica e Fisica

Università Cattolica del Sacro Cuore

Via Dei Musei 41, I-25121 Brescia, ItAly

E-mail address: marco.squassina@dmf.unicatt.it

(Zhang Xia) Department of Mathematics

Harbin Institute of Technology

HARBIN 150001, P.R. CHINA

E-mail address: piecesummer1984@163.com 